\title{
Overview of magnetic structure induced by the TEXTOR-DED and the related transport
}

\author{
S.S. Abdullaev ${ }^{1}$, K.H. Finken ${ }^{1}$, M.W. Jakubowski ${ }^{1}$, a , S.V. Kasilov ${ }^{2}$, \\ M. Kobayashi ${ }^{1}$, D. Reiser ${ }^{1}$, D. Reiter ${ }^{1}$, A.M. Runov ${ }^{3}$ and R. Wolf ${ }^{1}$ \\ ${ }^{1}$ Institut für Plasmaphysik, Forschungszentrum Jülich GmbH, EURATOM-Association, \\ Trilateral Euregio Cluster, D-52425 Jülich, Germany \\ ${ }^{2}$ Institute of Plasma Physics, National Center 'Kharkov Institute of Physics and Technology', \\ 61108 Kharkov, Ukraine \\ ${ }^{3}$ Max-Planck Institut für Plasmaphysik, D-17491 Greifswald, Germany
}

Received 13 October 2002, accepted for publication 28 February 2003

Published 15 April 2003

Online at stacks.iop.org/NF/43/299

\begin{abstract}
The dynamic ergodic divertor (DED), a new concept of the ergodic divertor, is presently installed for the TEXTOR tokamak. Beside the conventional ergodic divertor operation the DED also permits the operation with a rotating magnetic field which allows, in particular, to broaden the heat deposition pattern on the divertor plates. Since its first proposal of the DED in 1996 the structure of magnetic field, especially, the onset of ergodic zone of field lines and related transport in the DED-operation has been extensively studied using different theoretical and numerical methods. New methods to study the magnetic field, in particular, the field line mapping have been developed. The presentation gives the overview of the studies on the structure of magnetic field in the DED, the formation of the ergodic and laminar zones of field lines at the plasma edge. It also includes studies on the modelling efforts of the transport of heat and particles in the ergodic and laminar zones.
\end{abstract}

PACS numbers: 52.55.Dy, 52.55.Fa, 52.55.Rk

\section{Introduction}

The concept of the ergodic divertor has been introduced for the control of the plasma edge by creating ergodic magnetic field lines there by the external coils [1-3]. A new tool for the control of the plasma edge, the dynamic ergodic divertor (DED) [4], has been presently installed for the TEXTOR tokamak. Beside the conventional concept of the ergodic divertor implemented in the tokamaks TEXT [5,6], Tore-Supra [7-9, 11-13] and other fusion devices [14] the DED also permits the operation with a rotating magnetic field which allows, in particular, to broaden the heat deposition pattern on the divertor plates and possible, to impose the plasma rotation.

The DED is technically implemented by a quadruple set of 16 helical conductors (and two compensation coils) installed on the inboard side of the vessel at $r=0.535 \mathrm{~m}$ and covering the poloidal section of $\Delta \theta=70^{\circ}\left(\Delta \theta=80^{\circ}\right.$ with compensation coils). They are aligned parallel to the magnetic field lines at the plasma edge nearby a rational $q=3$ magnetic surface and optimized for the reference conditions $\beta_{\mathrm{pol}}=1$ and $r_{q=3} \approx 0.43 \mathrm{~m}$. The resonant $m: n$ perturbation field

a Also at: Institute of Physics, University of Opole, 45-052 Opolo, Poland. structure with the poloidal, $m(10 \leqslant m \leqslant 14)$ and toroidal, $n=4$, modes has been selected as the standard operational regime. This is achieved by a certain distribution of currents on coils. In the standard operational regime the perturbation field weakly penetrates into the plasma edge due to its fast radial decay. Much deeper penetration of the perturbation field can be achieved by the additional perturbation field structures with the toroidal modes $n=2$ and $n=1$ created by changing the current distribution on coils.

Due to the fact that particles predominantly follow the magnetic field the energy and particle transport in the ergodic zone depends significantly on the topology of the perturbed magnetic field and the structure of the field lines. These issues were the first important step in studying the DED operation. Since its first proposal in 1995 [15] the structure of magnetic field, especially, the onset of ergodic zone of field lines has been extensively studied using different theoretical and numerical methods [16-33]. A new method to study Hamiltonian systems, in particular, the field line mapping have been also developed to study the chaotic magnetic field lines $[28,34,35]$.

The study of ergodization of magnetic field lines by the external magnetic perturbations is an integral part of the 
more general problem of a magnetic stochasticity, i.e. the problem of destruction of magnetic surfaces under influence of symmetry breaking magnetic perturbations. This problem has been an important subject studied in a magnetically confined fusion devices since early 1960s as a mechanism which destroys the confinement of plasma [36-38]. Since the magnetic field lines are equivalent to the $1+\frac{1}{2}$ degrees of freedom Hamiltonian systems (see, e.g. [39,40]) the problem is fundamentally related to the dynamics of Hamiltonian systems under time-periodic perturbations [41,42].

Methods to study the perturbation field structure and its statistical properties in a toroidal system have been mostly based on the numerical codes [10-12, 16-19, 26]. The traditional approach to study the formation of the ergodic zone of field lines and their statistical properties are based, first, on the calculation of the spatial spectra of the perturbation magnetic field using the numerical codes, and, second, on the estimation of onset of magnetic stochasticity using the qualitative Chirikov's criteria of overlapping of magnetic islands.

The quantitative approach to the problem which reveals fine details of the ergodic zone at the plasma edge has been mainly based on field line tracing codes [12, 17, 24, 26]. Particularly, it allowed one to study the details of the so-called laminar zone, i.e. the outer region of the ergodic zone with the short wall to wall connection lengths. However, the field line tracing codes require huge computational times to study the properties of the ergodic and laminar zones. Moreover, since these codes, in principal, are not flux-preserving they lose the accuracy in the ergodic zone in a few poloidal turns [17].

For these reasons the computationally more effective mapping methods to study the ergodic zones have been proposed. The first simple mapping model to describe the formation of the ergodic zone in a cylindrical model of tokamak has been proposed by Martin and Taylor in [43]. This map has been applied in [44] to calculate the Lyapunov exponents and diffusion coefficients of field lines, and it has been further generalized in [45-47]. More realistic and generic model mappings, namely, the perturbed twist maps [42], for the magnetic field lines in ergodic divertors have been proposed in $[23,25,48,49]$.

The perturbed twist maps have been also previously used to describe the destruction of magnetic surfaces and diffusion of field lines in tokamaks [50,51], in stellarators [52,53], in a reversed field pinch $[54,55]$.

The flux-preserving mappings have been also used to describe the field lines in poloidal divertor tokamaks. Particularly, in [56-59] the specific algebraic maps, so-called tokamak divertor maps, have been proposed to describe the properties of field lines near the X-point and heat deposition patterns on the divertor plates. More general approach to these problems has been proposed in [60-62] using the separatrix mappings. The latter maps, unlike the divertor tokamak maps, are derived from the magnetic field line equations, and therefore, they can be applied to describe more realistic tokamak magnetic configurations.

There are several difficulties in applications of the perturbed twist maps to describe the field lines in realistic ergodic divertor tokamaks. Since the maps are not rigorously derived from the field line equations, in most cases the relation between mapping variables and corresponding variables in field line equations is not clear. Moreover, the method of derivation of the perturbed twist maps does not allow to estimate the accuracy of maps.

A rigorous method to derive symplectic maps from the continuous Hamiltonian equations, recently developed $[34,35]$, does not encounter such difficulties. The method based on the canonical transformation of variables and the perturbation theory allows one to construct the symplectic maps for generic Hamiltonian systems. These maps with large time steps comparable with the characteristic timescale of the system (perturbation period) have the same accuracy as the conventional symplectic integration methods with integration steps of two or three orders smaller. It has been successfully applied to study the formation of the ergodic and the laminar zone, field line diffusivity, and fractal properties of field lines in the DED of the TEXTOR [27, 28, 30,32].

The ergodic zone created at the plasma boundary modifies the heat and particle load patterns onto wall materials. The study of these processes is an important topic for the DED operation of the TEXTOR. The problem of heat and particle transport in a tokamak with chaotic field lines has been studied since a pioneering work of Rochester and Rosenbluth [63]. The radial diffusion of chaotic field lines acts as an addition to the usual perpendicular energy transport whereby increasing the radial transport through the ergodic zone due to large transport along the field lines [11]. The transport in fully developed ergodic regions of the magnetic field lines can be described by the quasilinear theory. It predicts the flat temperature profile at the plasma edge due to the large effective transverse diffusion in the ergodic zone $[64,65]$. However, the quasilinear theory was not able to describe the small-scale temperature structures in the ergodic zone measured in the experiments on the tokamaks TEXT, Tore Supra and CSTN-II $[6,11,66]$ : it has been observed the stationary (quasi) periodic radial, poloidal and toroidal structures with the magnitude of oscillations about $5-20 \mathrm{eV}$. Failure of the quasilinear theory to describe these features is due to the fact that in a real ergodic divertor operation the ergodic zone is not fully developed: it consists of remnants of magnetic islands inside the ergodic zone as well as the laminar zone near the divertor coils. In order to study this phenomenon and other features of temperature profile in the ergodic zone of Tore-Supra the three-dimensional non-linear transport model have been developed in [67-69]. The other transport models to describe the temperature profiles and the heat deposition patterns onto the divertor target plates in the DED of the TEXTOR have been also developed [24, 26, 70-77]. The first approach, a finite element method (FEM), is based on the idea that areas with relatively short connection lengths would play a predominant role in the transport at the edge region. In the numerical scheme these areas are treated separately like a scrape-off-layer (SOL), while the areas with long connection lengths are approximated as ergodic region characterized with the effective cross-field transport coefficients. The second method, a more powerful three-dimensional Monte-Carlo approach to the problem has been developed in $[76,77]$. The main advantage of the method is that it does not require any division of the ergodic zone into areas with different connection lengths. It treats the partially chaotic ergodic zone 
with remnants of islands as well as the regions with the intact magnetic surfaces in a similar way.

This paper is intended to review the main results obtained in studies of the magnetic field structure, formation and properties of the stochastic zone, and field line diffusivity in the DED of the TEXTOR. It also includes the results from modelling the heat transport in the ergodic zone of the TEXTOR-DED. It consists of four sections and conclusions. In section 2 we describe the DED coils, features of perturbed magnetic field and methods to study magnetic field lines in a tokamak under such a magnetic perturbation. The formation and structure of the ergodic zone and statistical properties of chaotic field lines are discussed in section 3. Studies on the energy and heat transport at the plasma periphery with the stochastic magnetic field lines and their application to the TEXTOR-DED are reviewed in section 4. Summary and conclusions are given in section 5 .

\section{Structure of magnetic perturbations}

In this section we describe configurational schemes of DED perturbation coils in different operational regimes, the main features of the magnetic field created by these coils. Methods of description of magnetic field lines in a tokamak in the presence of magnetic perturbations, particularly, Hamiltonian field line equations and a new mapping method to study them are also presented.

\subsection{The DED coils}

The set of divertor coils designed to create the resonant magnetic perturbations at the plasma edge consists of 16 helical coils each winding once around the torus starting at a toroidal, $\varphi_{j}=j \pi / 8$, and a poloidal angle $\theta_{j}=\pi-\theta_{\mathrm{c}}$ and ending after one toroidal turn at $\theta_{j}=\pi+\theta_{\mathrm{c}}$, where $j(j=1, \ldots, 16)$ stands for a coil number. They are located on the high field side (HFS) of the torus of minor radius $r_{\mathrm{c}}$ with a poloidal extension $\Delta \theta_{\mathrm{c}}=2 \theta_{\mathrm{c}} \approx 72^{\circ}$. A schematic view of the coil positions on the $(\varphi, \theta)$-plane is shown in figure $1(a)$.

Due to difficulties of technical implementation such a coil configuration of the coils are bundled in four quadruples and two additional (compensations) coils are added to compensate a net poloidal magnetic field that resulted from such a bundling [17]. The sketch of the technically implemented DED coils is shown in figure $1(b)$.

There are three operational regimes of the DED which are intended to create the resonant $m: n$ magnetic perturbations with the main $12: 4,6: 2$, and $3: 1$ resonances, respectively. In the standard DED operation $(n=4)$, the current distribution on the coils is given by

$$
I_{j}=I_{\mathrm{c}} \sin \left(\frac{\pi j}{2}+\Omega t\right) \quad(j=1, \ldots, 16),
$$

where $I_{\mathrm{c}}$ is a current amplitude $\left(I_{\mathrm{c}} \leqslant 15 \mathrm{kA}\right)$, and $\Omega$ is a rotation frequency of the perturbed field .

The $n=2$ mode regime. The current distribution (1) creates a perturbation field with a strong radial decay $B_{r} \propto$ $\left(r / r_{\mathrm{c}}\right)^{\alpha_{n=4}-1}$ with $\alpha_{n=4}=20$. Such a field weakly penetrates into the plasma, and it may be not sufficiently strong to form a
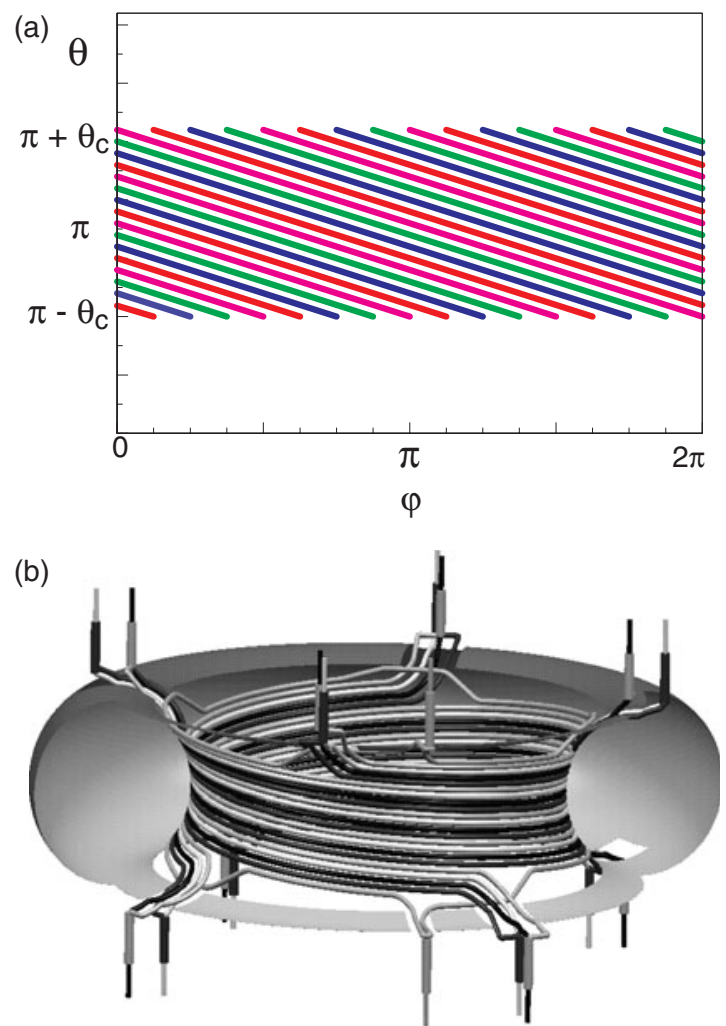

Figure 1. (a) Ideal DED coil configuration on the $(\varphi, \theta)$-plane; (b) sketch of the technically implemented DED coil configuration.

highly developed stochastic (ergodized) zone of field lines at the plasma edge. In order to overcome this it was proposed to switch the current distribution current on coils to the one with

$$
I_{j}=I_{\mathrm{c}} \sin \left(\frac{\pi j}{4}+\Omega t\right) \quad(j=1, \ldots, 16) .
$$

The distribution (2) creates the resonant $m: n$ magnetic perturbation with $n=2$, which has the twice smaller decaying exponent, $\alpha_{n=2}=\alpha_{n=4} / 2$ than the distribution (1).

The $n=1$ mode regime. More deeper penetration of the perturbed field into the plasma can be achieved by the current distribution $I_{j}=I_{\mathrm{c}} \sin (\pi j / 8+\Omega t)$. It is foreseen to use this mode regime to create the large size islands at the plasma edge to study MHD mode activities.

The mixture of $n=4$ and $n=2$ modes. A deep penetration of the perturbation field may provoke an excessive transport of energy and particles inside the core region or may even cause disruptions. To avoid such a possible scenario it is foreseen to use a superposition of the $n=4$ mode (1) with the $n=2$ mode (2) at arbitrary amplitudes. The current distribution on individual coils $I_{j}$ for such a combination is determined by

$$
\begin{aligned}
I_{j} & =(1-p) I_{\mathrm{c}} \sin \left(\frac{\pi j}{4}+\Omega t\right)+p I_{\mathrm{c}} \sin \left(\frac{\pi j}{2}+\Omega t\right) \\
& =I_{j 0} \sin \left(\alpha_{j}+\Omega t\right),
\end{aligned}
$$

where $I_{j 0}$ and $\alpha_{j}$ are the current amplitude and phase, respectively, in the coil $j$, and $p(0 \leqslant p \leqslant 1)$ is the relative contribution of the $n=2$ mode. For a given $p$ one can calculate the values of $I_{j 0}$ and $\alpha_{j}[30]$. 


\subsection{Magnetic perturbations}

The coil systems (1) and (2) create the magnetic perturbations at plasma periphery localized on the HFS of the torus and radially decaying toward inside the plasma $[17,28,30]$. It is mainly determined by the toroidal component of the vector potential

$$
A_{\varphi}(r, \theta, \varphi)=\sum_{m} A_{m n}(r, \theta) \cos (m \theta-n \varphi-\Omega t),
$$

where $r$ is a minor radial coordinate, $\theta$ and $\varphi$ are poloidal and toroidal angles. For large aspect ratio tokamaks, $R_{0} / a \gg 1$, where $R_{0}$ is a major radius of the torus, $a$ is a minor radius of the plasma, the Fourier coefficients $A_{m}(r)$ can be presented in the asymptotical form (see $[28,30]$ ):

$$
A_{m n}(r, \theta) \approx \frac{B_{\mathrm{c}} r_{\mathrm{c}} g_{m}}{\sqrt{1+r \cos \theta / R_{0}}}\left(\frac{r}{r_{\mathrm{c}}}\right)^{m},
$$

where $B_{\mathrm{c}}=2 \mu_{0} I_{\mathrm{c}} n / \Delta \theta_{\mathrm{c}} r_{\mathrm{c}}$ is a characteristic strength of the magnetic perturbation determined by the divertor current $I_{\mathrm{c}}$, the minor radius of coils $r_{\mathrm{c}}$, and the poloidal angular extension of the coil set $\Delta \theta_{\mathrm{c}}$. The quantity

$$
g_{m}=(-1)^{m+m_{\mathrm{c}}} \frac{\sin \left[\left(m-m_{\mathrm{c}}\right) \Delta \theta_{\mathrm{c}} / 2\right]}{\pi m\left(m-m_{\mathrm{c}}\right)}
$$

describes the poloidal spectrum of magnetic perturbations formed due to the finite poloidal extension of the coil set. The central mode number $m_{\mathrm{c}}$ is determined by $\Delta \theta_{\mathrm{c}}$ and the toroidal mode number $n: m_{\mathrm{c}}=2 \pi n / \Delta \theta$. The poloidal, $m$, spectrum of perturbation $A_{m}$ is localized near the central mode $m_{\mathrm{c}}$. The perturbed field has the toroidal mode $n=4$ and possesses a strong radial decay $A_{\varphi} \propto r^{m_{\mathrm{c}}}\left(m_{\mathrm{c}} \approx 20\right)$. The factor $1 / \sqrt{1+r \cos \theta / R_{0}}$ in (5) corresponds to the first order toroidal corrections.

The radial component of the perturbed magnetic field $B_{r}(\theta, \varphi)=r^{-1} \partial A_{\varphi} / \partial \theta$ on the $(\theta, \varphi)$ plane is shown in figure 2 for the standard DED operation $(n=4)$ and the ideal coil configuration. The TEXTOR-DED parameters are chosen as: $R_{0}=175 \mathrm{~cm}, r_{\mathrm{c}}=53.25 \mathrm{~cm}$, the current $I_{\mathrm{c}}=15 \mathrm{kA}$, $\Delta \theta_{\mathrm{c}}=2 \pi / 5$. A characteristic strength of the perturbed field $B_{\mathrm{c}}=2.251 \times 10^{3} \mathrm{G}$. The perturbed field profiles for the technically implemented coil configuration are calculated in [17].

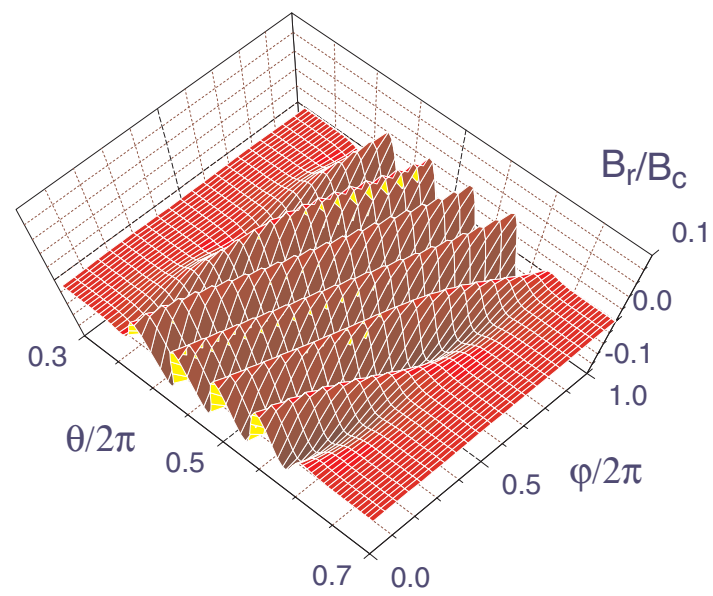

Figure 2. Radial component of the perturbed magnetic field $B_{r}$ in the $(\theta, \varphi)$-plane at the radial distance $r=46 \mathrm{~cm}$.

\subsection{Hamiltonian field line equations}

The Hamiltonian approach is the most natural and convenient method to study the stochastization of magnetic field lines. The Hamiltonian formulation of field line equations allows one to study directly the formation of the ergodic zone using, both the qualitative Chirikov's criteria and the symplectic mapping method.

The magnetic field lines are trajectories $x(\tau)=$ $(r(\tau), \theta(\tau), \varphi(\tau))$ determined by the equation: $\mathrm{d} \boldsymbol{x} / \mathrm{d} \tau=$ $\boldsymbol{B} /|\boldsymbol{B}|$, where $\tau$ is a length along a field line. This equation can be formulated in Hamiltonian form by introducing a coordinate system $(\psi, \vartheta, \varphi)$, where $\vartheta$ is a (so-called intrinsic) poloidal angle, and $2 \pi \psi$ is the amount of the toroidal magnetic flux enclosed by a magnetic surface of constant $\psi$. The spatial coordinates of field lines are unique functions of new coordinates, i.e. $\boldsymbol{x}=\boldsymbol{x}(\psi, \vartheta, \varphi)$. In a new coordinate system the divergence-free magnetic field can be presented in the so-called canonical form [39]: $\boldsymbol{B}=\nabla \psi \times \nabla \vartheta+\nabla \varphi \times \nabla \psi_{\mathrm{p}}$. The function $\psi_{\mathrm{p}}=\psi_{\mathrm{p}}(\psi, \vartheta, \varphi)$ is Hamiltonian function in the field lines equations:

$$
\frac{\mathrm{d} \vartheta}{\mathrm{d} \varphi}=\frac{\partial \psi_{\mathrm{p}}}{\partial \psi}, \quad \frac{\mathrm{d} \psi}{\mathrm{d} \varphi}=-\frac{\partial \psi_{\mathrm{p}}}{\partial \vartheta} .
$$

For the tokamak equilibrium magnetic field $\boldsymbol{B}$ with the nested magnetic surfaces the Hamiltonian $\psi_{\mathrm{p}}$ is a function of only the toroidal flux $\psi$ and it is determined only by the safety factor $q(\psi)$, i.e. $\psi_{\mathrm{p}}^{(0)}(\psi)=\int \mathrm{d} \psi / q(\psi)$. Then the magnetic field lines are straight, $\vartheta=\varphi / q(\psi)$, and lie on the magnetic surface of constant $\psi=$ const.

For any non-axisymmetric magnetic perturbations in tokamaks the Hamiltonian functions can be presented in the form

$$
\psi_{\mathrm{p}}(\psi, \vartheta, \varphi)=\psi_{\mathrm{p}}^{(0)}(\psi)+\epsilon \psi_{\mathrm{p}}^{(1)}(\psi, \vartheta, \varphi),
$$

where $\epsilon$ is a dimensionless parameter of perturbation, and $\psi_{\mathrm{p}}^{(1)}(\psi, \vartheta, \varphi)$ is a perturbed part of the Hamiltonian. The latter is a periodic function of the poloidal, $\vartheta$, and toroidal, $\varphi$ angles, and may be presented as a Fourier series

$$
\psi_{\mathrm{p}}^{(1)}(\psi, \vartheta, \varphi)=\sum_{m n} h_{m n}(\psi) \cos \left(m \vartheta-n \varphi-\chi_{m n}\right),
$$

where the terms $\epsilon h_{m n}(\psi) \cos \left(m \vartheta-n \varphi-\chi_{m n}\right)$ correspond to the resonant magnetic perturbations, and $\chi_{m n}$ are phases. For the magnetic perturbations (4) $\chi_{m n}=\Omega t$ and the perturbation parameter $\epsilon$ is determined by the ratio of the characteristic strength of the perturbed magnetic field, $B_{\mathrm{c}}$, to the toroidal magnetic field, $B_{\varphi}$, i.e. $\epsilon=B_{\mathrm{c}} / B_{\varphi}$.

One should note that the poloidal spectrum of perturbations, $h_{m n}(\psi)$, in (8) is different from the spectrum of magnetic field perturbations $A_{m}$ (5). The spectrum of $h_{m n}(\psi)$ depends on the relation between the spatial coordinates $(r, \theta)$ and the magnetic coordinates $(\psi, \vartheta)$ of the equilibrium magnetic configuration. The relation between $h_{m n}(\psi)$ and $A_{m}$ will be studied in section 2.5 .

\subsection{Mapping method to integrate field line equations}

A direct integration of Hamiltonian equations (6) using the ordinary integration schemes like Runge-Kutta methods, in 
principle, are not ideal for this purpose. The numerical approximation to Hamiltonian systems obtained from these methods introduces non-Hamiltonian perturbations. This means that they do not conserve an area-preserving property of Hamiltonian systems, which is equivalent to a flux-preserving property of the magnetic field. It may lead to completely different long-term behaviour of the system. From the computational point of view they require long computational times.

To study Hamiltonian field line equations a new symplectic mapping method has been developed [28, 34, 35]. The method is applicable for generic Hamiltonian systems of type (6)-(8). The flux-preserving maps are constructed using the methods of the Hamilton-Jacobi theory and the classical perturbation theory. Particularly, a generating function associated with the map is found using the nonstationary perturbation theory. The maps run much faster than the standard integration methods of ordinary differential equations.

The mapping of field lines in tokamaks is constructed by the following way. Introduce poloidal sections $\varphi=\varphi_{k}=$ $(2 \pi / s) k,(k=0, \pm 1, \pm 2, \ldots)$, where $s(s \geqslant 1)$ is an integer number which stands for a number of map steps per one toroidal rotation along the torus. The mapping

$$
\left(\vartheta_{k+1}, \psi_{k+1}\right)=\hat{M}\left(\vartheta_{k}, \psi_{k}\right),
$$

relates the cross-section points, $\left(\vartheta_{k}, \psi_{k}\right)$, of the field line $(\vartheta(\varphi), \psi(\varphi))$ with the poloidal section $\varphi=\varphi_{k}$, with the ones $\left(\vartheta_{k+1}, \psi_{k+1}\right)$ at $\varphi=\varphi_{k+1}$. For the Hamiltonian system (6)-(8) the mapping is constructed in the following symmetric flux-preserving form

$$
\begin{gathered}
\Psi_{k}=\psi_{k}-\epsilon \frac{\partial S_{k}}{\partial \vartheta_{k}}, \quad \Theta_{k}=\vartheta_{k}+\epsilon \frac{\partial S_{k}}{\partial \Psi_{k}}, \\
\Psi_{k+1}=\Psi_{k}, \quad \Theta_{k+1}=\Theta_{k}+\frac{\varphi_{k+1}-\varphi_{k}}{q\left(\Psi_{k}\right)}, \\
\psi_{k+1}=\Psi_{k+1}+\epsilon \frac{\partial S_{k+1}}{\partial \vartheta_{k+1}}, \quad \vartheta_{k+1}=\Theta_{k}-\epsilon \frac{\partial S_{k+1}}{\partial \Psi_{k+1}},
\end{gathered}
$$

with the generating function $S_{k}=\left.S(\vartheta, \Psi, \varphi ; \epsilon)\right|_{\varphi=\varphi_{k}}$. The Poincaré map may be obtained by applying the map (9) $s$ times.

The generating function $S(\vartheta, \Psi, \varphi ; \epsilon)$ satisfies the Hamilton-Jacobi equation, the solutions of which are sought using the perturbation theory in the finite interval $\varphi_{k+1}<\varphi<\varphi_{k}$. In the first order of $\epsilon$ it is determined by

$$
\begin{aligned}
& S(\vartheta, \Psi, \varphi)=-\left(\varphi-\varphi_{0}\right) \sum_{m} h_{m n}(\Psi) \\
& \left.\quad \times\left[a\left(x_{m n}\right) \sin \phi_{m n}+b\left(x_{m n}\right) \cos \phi_{m n}\right)\right]+\mathrm{O}(\mu),
\end{aligned}
$$

where $\phi_{m n}=m \vartheta-n \varphi-\chi_{m n}, x_{m n}=(m / q(\Psi)-n)\left(\varphi-\varphi_{0}\right)$, $a(x)=(1-\cos x) / x$, and $b(x)=\sin x / x$. The free parameter $\varphi_{0}$ lies in the interval $\varphi_{k} \leqslant \varphi_{0} \leqslant \varphi_{k+1}$. The actual small expansion parameter of the generating function is $\mu=\epsilon\left(\varphi-\varphi_{0}\right)^{\delta} \ll 1,(\delta \geqslant 1)$, and the term $\mathrm{O}(\mu)$ in (13) stands for the corrections of order of $\mu$ and its higher orders. Therefore, the mapping can be applied systems with moderately large perturbation by taking the map time step sufficiently small.

The maps with large time steps comparable with the characteristic timescale of the system (e.g., a perturbation period) have the same accuracy as the standard symplectic integrator with integration steps of two or three orders smaller [35].

\subsection{Spectrum of magnetic perturbations in a toroidal system}

The behaviour of field lines mainly determined by the spectrum of the resonant magnetic perturbations in $(8), h_{m n}(\psi)$. In general, they depend on the vacuum magnetic perturbations (4), (5) and the equilibrium plasma configuration. They are determined by

$$
\begin{array}{r}
h_{m n}(\psi)=\operatorname{Re} \frac{1}{\left(2 \pi R_{0}\right)^{2}} \int_{0}^{2 \pi} \int_{0}^{2 \pi} R(x(\psi, \vartheta, \varphi)) \\
\quad \times A_{\varphi}(x(\psi, \vartheta, \varphi)) \exp (-\mathrm{i} m \vartheta+\mathrm{i} n \varphi) \mathrm{d} \vartheta \mathrm{d} \varphi,
\end{array}
$$

where $R(\boldsymbol{x})=R_{0}+r \cos \theta$. Since the perturbation is localized on the HFS for the large mode numbers $m$ equation (14) can be presented in the form $h_{m n}(\psi) \approx \sum_{m^{\prime}} S_{m m^{\prime}}(\psi) \bar{A}_{m^{\prime} n}$, where $\left.\bar{A}_{m^{\prime} n} \equiv R(\psi, \vartheta) A_{m^{\prime} n}(r(\psi, \vartheta), \theta(\psi, \vartheta))\right|_{\vartheta=\pi}$, i.e. the Fourier components (5) taken on the HFS. The transformation matrix $S_{m m^{\prime}}(\psi)$ is mainly determined by the integral:

$S_{m m^{\prime}}(\psi)=\frac{1}{2 \pi} \int_{0}^{2 \pi} \exp \left[-\mathrm{i} m \vartheta+\mathrm{i} m^{\prime} \theta(\psi, \vartheta)\right] \mathrm{d} \vartheta$,

which is determined by the relation between the intrinsic coordinate $\vartheta$ and the poloidal angle $\theta$. The relation $\vartheta=\vartheta(\theta)$ depends on the plasma parameters, especially on the plasma $\beta_{\text {pol }}$. A typical dependence $\vartheta$ on $\theta$ for the different $\beta_{\text {pol }}$ is shown in figure 3 .

It was found that the matrix $S_{m m^{\prime}}(\psi)$ does not simply connect the neighbouring $m^{\prime}=m \pm 1$ modes, but it has general coherent features [28]. The asymptotical estimation of the integral (15) shows that

$$
S_{m m^{\prime}}(\psi) \approx \frac{(-1)^{m+m^{\prime}}}{\left(\beta_{3} m^{\prime} / 2\right)^{1 / 3}} \operatorname{Ai}\left(\frac{\beta_{1} m^{\prime}-m}{\left(\beta_{3} m^{\prime} / 2\right)^{1 / 3}}\right),
$$

where $\operatorname{Ai}(x)$ is the Airy function. The matrix $S_{m m^{\prime}}(\psi)$ depends on the first, $\beta_{1}=\mathrm{d} \theta /\left.\mathrm{d} \vartheta\right|_{\theta=\pi}$, (the pitch angle), and third, $\beta_{3}=$ $\mathrm{d}^{3} \theta /\left.\mathrm{d} \vartheta^{3}\right|_{\theta=\pi}$, derivatives of the poloidal geometrical angle, $\theta$, with respect to the intrinsic angle, $\vartheta$, respectively, taken on the HFS. The dependence of $S_{m m^{\prime}}(\psi)$ on the mode number $m^{\prime}$ for the fixed $m=12$ at the last magnetic surface $r=a$ is plotted in figure 4. It shows that the main contribution to the fixed mode $m$ comes from the group of vacuum perturbation modes $m^{\prime}$ located near $m / \beta_{1} \approx m_{\mathrm{c}}=20$.

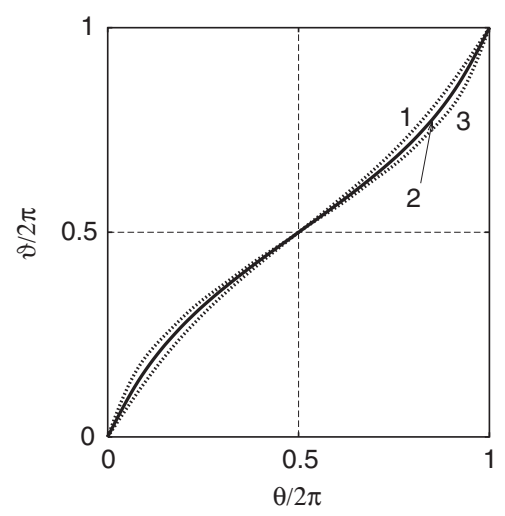

Figure 3. Dependence of the intrinsic angle $\vartheta$ on the poloidal angle $\theta$ for the different $\beta_{\mathrm{pol}}$ : curve 1 corresponds to $\beta_{\mathrm{pol}}=0$, curve 2-to $\beta_{\text {pol }}=1$, curve 3 -to $\beta_{\text {pol }}=2$. 


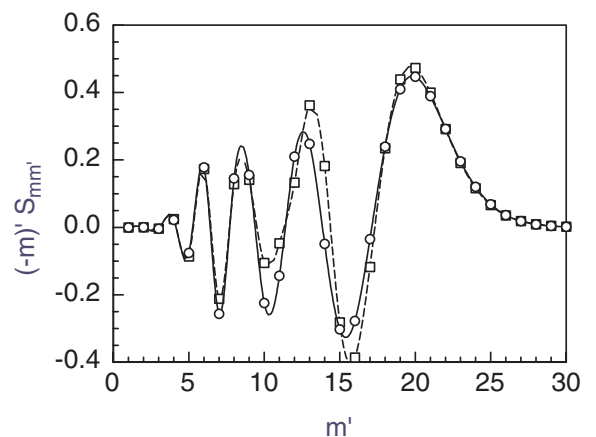

Figure 4. Dependence of the transformation matrix $S_{m m^{\prime}}$ on the vacuum perturbation mode $m^{\prime}$ at the fixed $m$. Solid curve corresponds to the exact formula (15), dashed curve- to the asymptotic formula (16).

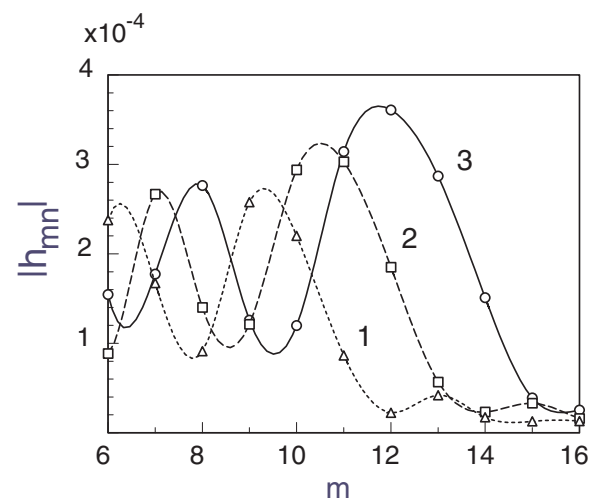

Figure 5. Spectrum $\left|h_{m n}\right|(n=4)$ for different values of $\beta_{\mathrm{pol}}$ : curve 1 corresponds to $\beta_{\text {pol }}=1.0$, curve 2 - to $\beta_{\text {pol }}=0.6$, curve 3 - to $\beta_{\text {pol }}=0.2$. Plasma current $I_{\mathrm{p}}=0.5 \mathrm{MA}$, toroidal field $B_{\varphi}=1.875 \mathrm{~T}$, divertor current $I_{\mathrm{c}}=15 \mathrm{kA}$.

Using (5) and (16), one can obtain asymptotical formula for the spectrum $h_{m n}$ for large mode numbers $m$ [28]:

$$
\begin{aligned}
& h_{m n}(\psi) \approx(-1)^{m+m_{\mathrm{c}}} \frac{r_{\mathrm{c}}}{R_{0}} \sqrt{1-\frac{r(\psi, \pi)}{R_{0}}} \\
& \times\left(\frac{r(\psi, \pi)}{r_{\mathrm{c}}}\right)^{m^{*}} \frac{\sin \left(m^{*}-m_{\mathrm{c}}\right) \Delta \theta_{\mathrm{c}} / 2}{\pi \beta_{1} m^{*}\left(m^{*}-m_{\mathrm{c}}\right)},
\end{aligned}
$$

where $m^{*}=\left(m+\left[m \beta_{3} / 2 \beta_{1}\right]^{1 / 3}\right) / \beta_{1}$. It describes the main features of the transformation of the spectrum of vacuum magnetic perturbations into ones in a toroidal plasma. The poloidal spectrum $h_{m n}$ (17) is located near the central mode $m_{\mathrm{c}}^{*} \approx m_{\mathrm{c}} \beta_{1}$ with the width $\Delta m \approx \pi \beta_{1} / \Delta \theta_{\mathrm{c}}$. These quantities are mainly determined by the parameter $\beta_{1}$, which depends on the plasma $\beta_{\text {pol }}$ as well as the flux coordinate $\psi$. Therefore one can control the spectrum $h_{m n}$ by simple varying $\beta_{\text {pol }}$. Figure 5 shows an example of such a variation of $h_{m n}$ at the resonant magnetic surface $\psi=\psi_{m n}(m=10, n=4)$.

\section{Properties of the ergodic and the laminar zones}

In this section we study the main features of the ergodic and laminar zones, fractal and statistical properties of chaotic field lines.

\subsection{Model of the equilibrium plasma}

We consider a model of the tokamak plasma equilibrium with nested, circular magnetic surfaces (see $[10,28,30]$ ). Let $\rho$ be a radius of the magnetic surface with the centre at $R_{\mathrm{p}}(\rho)$ outward shifted from the centre of the outmost magnetic surface of radius $a$ on the distance $\Delta(\rho)=R_{\mathrm{p}}(\rho)-R_{\mathrm{p}}(a)$ known as the Shafranov shift: $\Delta(\rho)=\left[R_{\mathrm{p}}(a)^{2}+(\Lambda+1)\left(a^{2}-\rho^{2}\right)\right]^{2}-R_{\mathrm{a}}$. Here $\Lambda=\beta_{\mathrm{pol}}+l_{\mathrm{i}} / 2-1$, where $\beta_{\mathrm{pol}}$ is a ratio of the plasma pressure to the pressure of the poloidal field, and $l_{\mathrm{i}}$ is the internal inductance. The safety factor $q(\rho)$ is determined by the plasma current $I_{\mathrm{p}}$ and the current of the toroidal system $I_{\varphi}[28,30]$ :

$$
q(\rho)=\frac{I_{\varphi}}{I_{\mathrm{p}}} \frac{\rho^{2}}{R_{\mathrm{p}}^{2}}\left(1+\frac{a_{2}}{2} \frac{\rho^{2}}{R_{\mathrm{p}}^{2}}+\frac{3 a_{4}}{8} \frac{\rho^{4}}{R_{\mathrm{p}}^{4}}+\cdots\right),
$$

where

$$
a_{m}=\sum_{k=0}^{m}(m-k+1) \Lambda^{k} .
$$

The toroidal flux $\psi$ (normalized to the $B_{\varphi} R_{0}^{2}$ ) is related the radius $\rho$ as

$$
\psi=1-\left(1-\frac{\rho^{2}}{R_{\mathrm{p}}^{2}}\right) \frac{1}{2} \approx \frac{\rho^{2}}{2 R_{0}} .
$$

For the numerical calculations we will use the following plasma parameters: the major radius $R_{0}=175 \mathrm{~cm}$, the plasma radius $a=46 \mathrm{~cm}$, the divertor plate $r_{\mathrm{d}}=49 \mathrm{~cm}$, the divertor current $I_{\mathrm{c}}=15 \mathrm{kA}$, the radial position of coils $r_{\mathrm{c}}=53.25 \mathrm{~cm}$.

\subsection{Onset of the ergodic and laminar zones}

The resonant magnetic perturbations $h_{m n}(\psi) \cos (m \vartheta-n \varphi+$ $\chi_{m n}$ ) in (8) strongly affect the field lines on the resonant magnetic surfaces $\psi=\psi_{m n}\left(q\left(\psi_{m n}\right)=m / n\right)$ forming the chain of magnetic islands with the width

$$
\Delta \psi_{m n}=4\left|\frac{\epsilon h_{m n}(\psi)}{\mathrm{d} q^{-1} / \mathrm{d} \psi}\right|^{1 / 2} .
$$

At certain level of the DED perturbation the isolated magnetic islands start to overlap and create the ergodic zone of field lines. At the standard operational regime the central mode number $m_{\mathrm{c}}^{*}$ may vary between 10 and 13 , and the ergodic zone is formed by overlapping of resonances in the interval: $m_{\mathrm{c}}^{*}-\Delta m / 2 \leqslant m \leqslant m_{\mathrm{c}}^{*}+\Delta m / 2$ at the fixed toroidal mode $n=4$. The structure and the degree of ergodization of field lines depend not only on the mutual radial positions of resonant magnetic surface $\psi_{m n}$ but also on how close they are located to the divertor coils at $r=r_{\mathrm{c}}$. This is because of the strong radial dependence of the magnetic perturbations (see equations (5) and (17)). By the outward shift of the resonant magnetic surfaces $\psi_{m n}$ one can increase the width of magnetic islands, and therefore the ergodization level. If the amplitude, $\epsilon h_{m n}$, of the outermost resonant surface $\psi_{m n}$ is sufficiently large it creates a bump on the outside of the island whereby it touches the divertor plate. This island dominates over inner islands and forms the region with the regular open field lines. This is partly responsible for the formation of the laminar zone, a zone field lines with very short wall to wall connection lengths. 


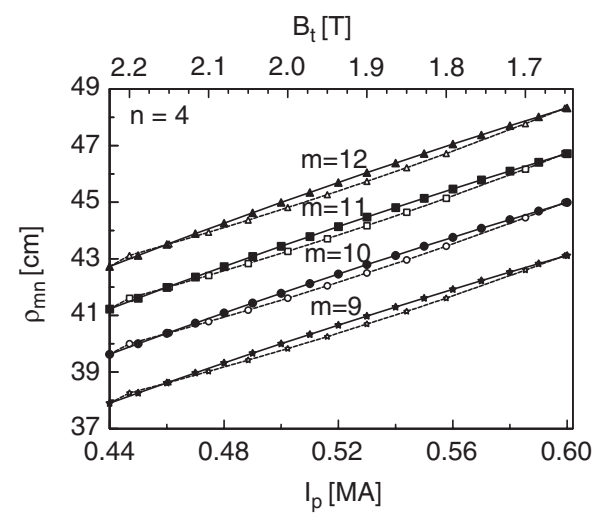

Figure 6. Radial positions of $\rho_{m n}$ vs $I_{\mathrm{p}}(-)$ at a fixed $B_{\varphi}=2.25 \mathrm{~T}$ and vs $B_{\varphi}(---)$ at the fixed $I_{\mathrm{p}}=0.44 \mathrm{MA}$.

There are several options to vary the operational regimes of the DED from the regimes with the predominantly ergodized field lines (with very long connection lengths) to the (laminar) one with the predominantly regular field lines with very short connection lengths. These options are: (i) regulation of the divertor current $I_{\mathrm{c}}$; (ii) variation of the plasma $\beta_{\mathrm{pol}}$; (iii) changing the radial positions of resonant magnetic surfaces $\psi_{m n}$. The first option does not produce a sufficient variation of the operation regime, especially for the higher $\beta_{\mathrm{pol}} \geqslant 1$ [30]. The lowering $\beta_{\text {pol }}$ may increase the ergodization level of field lines $[28,30]$, however, this option is not desirable. The third option, a shift of the radial positions of the resonant magnetic surfaces, $\psi_{m n}$, is the most convenient way to regulate the operational regime of the DED. It can be implemented by varying the plasma current $I_{\mathrm{p}}$ or the toroidal magnetic field $B_{\varphi}$ (through the main current of the magnetic system $I_{\mathrm{t}}$ ). In figure 6 the dependences of $\rho_{m n} \approx R_{0} \sqrt{2 \psi_{m n}}$ on the plasma current $I_{\mathrm{p}}$ (at the fixed value of the toroidal magnetic field $B_{\varphi}$ ) and on the toroidal field $B_{\varphi}$ (at the fixed value of the plasma current $I_{\mathrm{p}}$ ) are plotted.

\subsection{The ergodic and laminar zones}

The stochastization of field lines creates the region of open field lines at the plasma edge connecting wall to wall. This region may be roughly divided into two zones, the ergodic and the laminar zones. The zone of field lines with large connection lengths (roughly, more than six poloidal turns) can be defined as an ergodic zone, while the zone of field lines with a few poloidal turns defines a laminar zone. The variation of these zones by changing the plasma parameters, for example, the plasma current or the toroidal magnetic field, allows one to study the different regimes of the plasma edge extended from ergodic dominated edge to those similar to normal helical divertor structures.

First consider the variation the plasma regimes with the plasma $\beta_{\mathrm{pol}}$. In figure 7 Poincaré sections of field lines at the plasma edge for the three different $\beta_{\mathrm{pol}}$ at the fixed plasma current $I_{\mathrm{p}}=420 \mathrm{kA}$ are displayed: $(a) \beta_{\mathrm{pol}}=0 ;(b) \beta_{\mathrm{pol}}=1$; (c) $\beta_{\mathrm{pol}}=2$. One can see that the increase of $\beta_{\text {pol }}$ drastically changes the regime of field lines from the well-developed ergodic layer for $\beta_{\mathrm{pol}}=0$ to the regular case with slightly deformed magnetic surfaces for $\beta_{\mathrm{pol}}=2$.
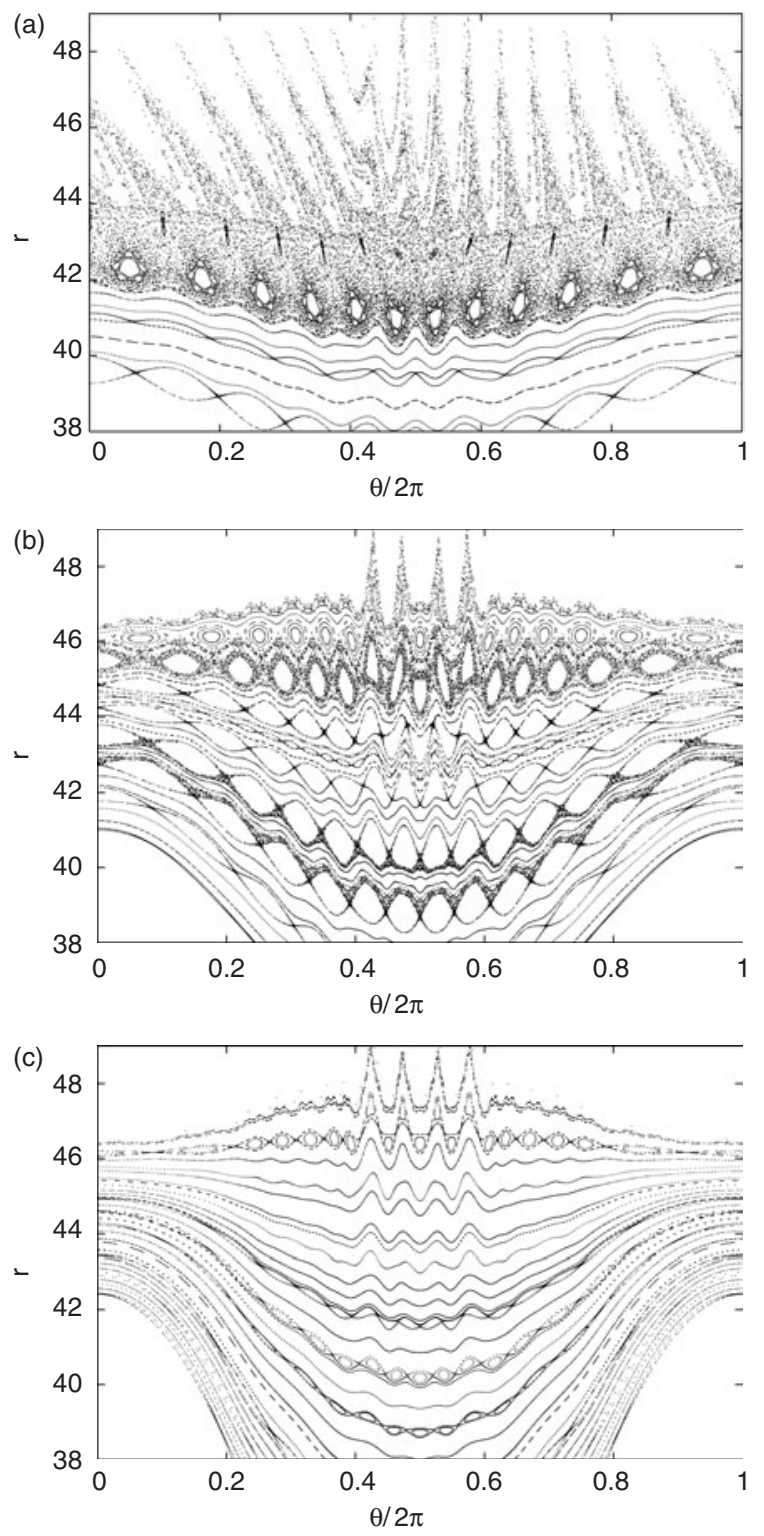

Figure 7. Poincaré sections of magnetic field lines in the $(r, \vartheta)$ : (a) $\beta_{\mathrm{pol}}=0 ;(b) \beta_{\mathrm{pol}}=1 ;(c) \beta_{\mathrm{pol}}=2$; the plasma current $I_{\mathrm{pol}}=420 \mathrm{kA}$.

In order to enhance the ergodization level for the plasma with the higher $\beta_{\mathrm{pol}}$ one can increase the plasma current $I_{\mathrm{p}}$. A typical dependence of the ergodization level on $I_{\mathrm{p}}$ is shown in figure 8 for the plasma currents: (a) $I_{\mathrm{p}}=460 \mathrm{kA}$, (b) $I_{\mathrm{p}}=520 \mathrm{kA}$, and $(c) I_{\mathrm{p}}=580 \mathrm{kA}$. Figure $8(a)$ displays the ergodic zone dominated regime, while figure $8(c)$ shows the laminar zone dominated regime. The large white areas in figure $8(c)$ correspond to field lines with very short connection lengths. One can see that the ergodic zone is substantially decreased (approximately from $5 \mathrm{~cm}$ to several $\mathrm{mm}$ on the HFS) when the plasma current is varied from 460 to $580 \mathrm{kA}$, while the laminar zone is increased approximately $2 \mathrm{~cm}$.

The chaotic field lines in the ergodic layer contribute to the radial energy and particle transport at the plasma edge in addition to the usual perpendicular energy transport. The transport due to chaotic field lines can be described by the field line diffusion coefficients $D_{\mathrm{FL}}$. In the quasilinear 

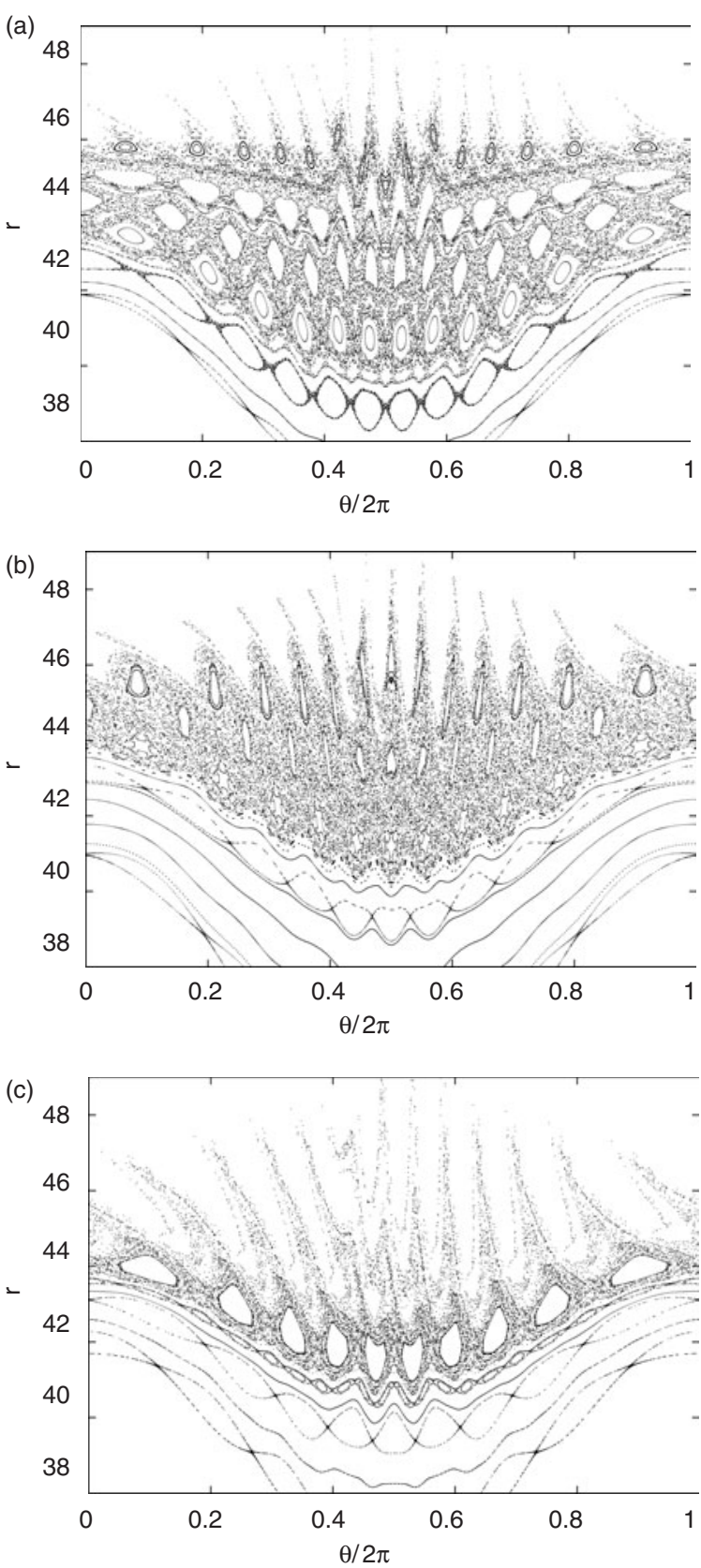

Figure 8. The same as in figure 7, but for the different plasma current: (a) $I_{\mathrm{p}}=460 \mathrm{kA}$; (b) $I_{\mathrm{p}}=520 \mathrm{kA}$; $(c) I_{\mathrm{p}}=580 \mathrm{kA}$. The plasma $\beta_{\text {pol }}=1$.

approximation the diffusion coefficient for the Hamiltonian system (6)-(8) is determined by [42]:

$$
D_{\mathrm{Q}}=\pi R_{0} \epsilon^{2} \sum_{m n}\left|m h_{m n}(\psi)\right|^{2} \delta\left(\frac{m}{q(\psi)}-n\right) .
$$

It coincides with the traditionally used quasilinear formula for $D_{\mathrm{FL}}$ if $m \epsilon h_{m n}$ is replaced by the coefficients $B_{m n}$ in a Fourier expansion of the perturbed field $B_{r}$ [66].

The field line diffusion in the ergodic zone can be also studied numerically by calculating the second order radial displacement moments

$$
\sigma_{\rho_{0}}(l)=\left\langle(\rho(l)-\langle\rho\rangle)^{2}\right\rangle,
$$
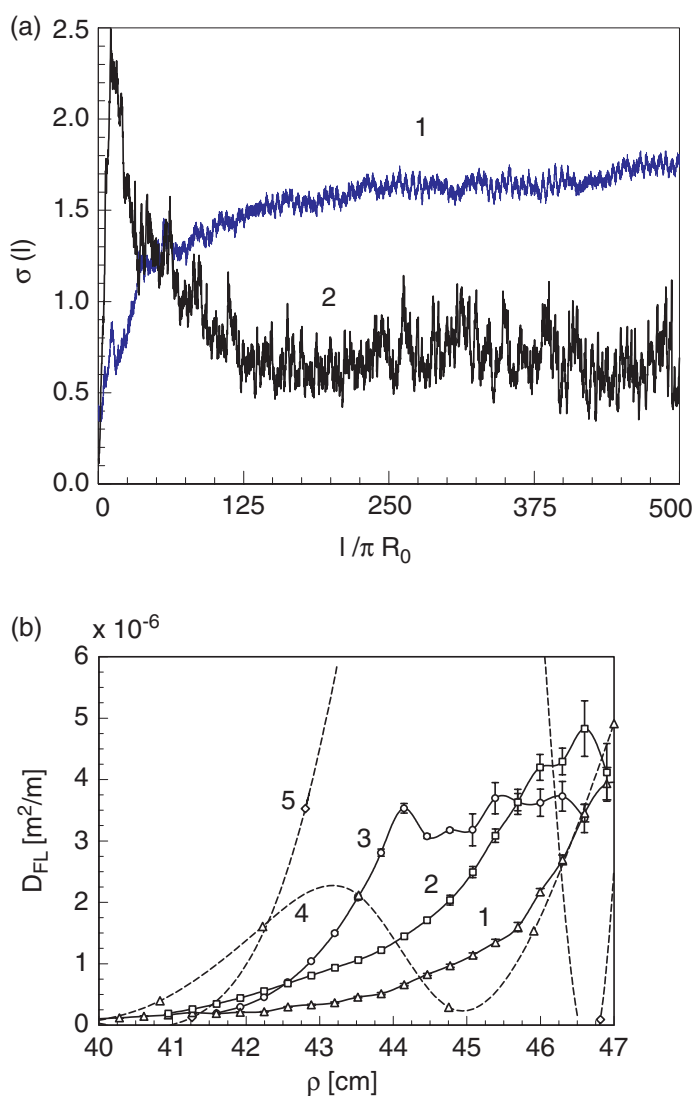

Figure 9. (a) A typical behaviour of the mean square radial displacement $\sigma(l)$ vs the length $l$ along the toroidal angle $\varphi$ for the two case: curve 1 describes the case when field lines do not leave the ergodic zone; curve 2-when field lines hit the divertor plates. (b) Radial profiles of local field line diffusion coefficients $D_{\mathrm{FL}}$ for different plasma currents: curve 1 corresponds to $I_{\mathrm{p}}=460 \mathrm{kA}$, curve 2 - to $I_{\mathrm{p}}=520 \mathrm{kA}$, curve 3 - to $I_{\mathrm{p}}=580 \mathrm{kA}$. Quasilinear diffusion coefficients $D_{\mathrm{Q}}$ are plotted by dashed curves: 4 -for $I_{\mathrm{p}}=460 \mathrm{kA}$, and 5-for $I_{\mathrm{p}}=520 \mathrm{kA}$. The plasma $\beta_{\mathrm{pol}}=1$.

where $\langle(\cdots)\rangle$ means averaging over a set of field lines with initial angle $\theta$ being uniformly distributed on the magnetic surface $\rho=\rho_{0}(\theta)$, and $l$ stands for the distance along field lines. A typical dependence $\sigma_{\rho_{0}}(l)$ on $l$ in the ergodic zone is shown in figure $9(a)$ for the two cases: curve 1 describes the case of the closed ergodic zone; curve 2 corresponds to the laminar zone dominated case. In the first case the field lines are confined in the ergodic zone and they do not reach the divertor plate, while in the second case the field lines are open and they reach the divertor plate after a certain number of poloidal turns. It has the following features: $\sigma_{\rho_{0}}(l)$ grows with $l$ up to a certain distance, when field lines reach the boundaries of the ergodic zone, and then it converges to a constant value (curve 1 figure $9(a)$ ) in or it decreases when field lines leave the ergodic zone hitting the divertor plates (curve 2 figure $9(a)$ ).

In this situation one cannot introduce a global diffusion coefficient $D=\sigma_{\rho_{0}}(l) / 2 l,(l \rightarrow \infty)$ as in the case of unlimited stochastic domain. However, one can introduce a local diffusion coefficient $D_{\mathrm{FL}}\left(\rho_{0}\right)$ which is valid for the initial linear growth regime of $\sigma_{\rho_{0}}(l)$ with $l$ [28]. Typical profiles of $D_{\mathrm{FL}}(\rho)$ as a function of magnetic surface radius $\rho$ are presented in figure $9(b)$ for the different plasma currents. The corresponding quasilinear diffusion coefficients $D_{\mathrm{Q}}(20)$ 
are also plotted in this figure. One can see that $D_{\mathrm{FL}}$ grows with $\rho$ monotonically up to the certain radius $\rho_{1}$ then it decays in the laminar zone $\rho>\rho_{1}$ where the contribution of field lines in (21) is decreased because of very short connection lengths. The radius $\rho_{1}$ characterizes the inner boundary of the laminar zone. The width of the laminar zone grows with the plasma current $I_{\mathrm{p}}$.

Usually the quasilinear diffusion coefficients overestimate the diffusion transport rate in the DED ergodic zone. The quasilinear theory is not valid in this case since the ergodic zone is formed by overlapping only a few neighbouring magnetic islands.

One should note that the local and quasilinear diffusion coefficients do not completely describe the transport processes in the ergodic zone. They are valid only for the highly developed ergodic zone. The real ergodic zone at the plasma edge is not well-developed, and it consists of areas with the remnants of magnetic islands as well as areas with almost regular field lines with short connection lengths (the laminar zone). The transport processes in such an ergodic zone cannot be simply described by the quasilinear theory.

\subsection{Fractal properties of field lines}

The magnetic field lines in the ergodic zone at the plasma periphery can be viewed as a chaotic scattering system (see [78] and references therein) whereby field lines enter into the plasma from the wall and leave it when hitting the wall after a certain number of toroidal (or poloidal) turns [32]. The length of field lines inside the plasma region is very sensitive to their initial coordinates: a tiny change of the input conditions can produce drastic changes in the length of field lines.

In chaotic scattering systems a trajectory may leave the system in one of several different ways. The space of initial coordinates corresponding to the various exit ways are separated by a boundary which may be a fractal. The set of initial conditions for which trajectories leave the system in a particular way is called the basin of particular mode.

Since the DED perturbations are localized on the HFS of the torus the field lines enter the plasma and leave it on the HFS making almost full poloidal turns. Therefore, it is convenient to classify the field lines by the number of poloidal turns $N_{\mathrm{p}}$ (see figure 12 $(a)$ where the example of a field line with the two poloidal turns is shown). Spatial structures of basins belonging to the different $N_{\mathrm{p}}$ give fine details of the ergodic and the laminar zone which cannot be revealed by Poincaré sections. For this purpose one can use the contour plots of $N_{\mathrm{p}}$ (or so-called laminar plots) within the plasma edge and on the divertor target plates [24,32].

The example of the contour plot of $N_{\mathrm{p}}$ in the $(\vartheta, \psi)$-plane for the plasma current $I_{\mathrm{p}}=580 \mathrm{kA}$ is shown in figure 10: (a) shows the plot on the HFS, and $(b)$ shows the expanded view of the rectangular area in $(a)$. The vertical axis is normalized to the value of the toroidal flux, $\psi_{\mathrm{a}}$, through last unperturbed magnetic surface of radius $a$. In figure $10(a)$ the basins corresponding up to the six poloidal turns are shown, while in figure $10(b)$ - up to $N_{\mathrm{p}} \leqslant 10$. The basins corresponding to the field lines with different $N_{\mathrm{p}}$ are coloured according the colour-bar. The darkest blue basin corresponds to shortest field lines with connection lengths less than one poloidal turns.
The darkest red area describes the basins with more than six poloidal turns in figure $10(a)$, and more than ten in figure $10(b)$. The boundaries between these basins are not resolved.

One of the specific features of the laminar zone revealed by the laminar plots is existence of long dark elongated stripes (so-called 'fingers') extended up to the divertor plate. They consist of basins with different $N_{\mathrm{p}}$ and connect the ergodic zone with the divertor target plates. The structure of the stripes has a complicated fractal nature. The magnification of the finger area shows the self-similar layered areas with the different $N_{\mathrm{p}}$ with fractal boundaries (see figure 10(b) and [32] for details).

Since particles predominantly follow the magnetic field lines heat and particle deposition patterns on the divertor plates will be mainly determined by magnetic footprints on these plates. The examples of magnetic footprints on the $(\varphi, \theta)$-plane of the divertor target plate for the two different plasma currents are shown in figure 11: (a) $I_{\mathrm{p}}=460 \mathrm{kA}$; (b) $I_{\mathrm{p}}=580 \mathrm{kA}$. Because of the four-fold symmetry along the toroidal direction only one quarter of magnetic footprints are presented. One can see from figure 11 that magnetic footprints consist of four pairs of narrow helical stripes. The field lines coming from the plasma hit the target plates along these stripes. (Dark blue areas correspond to the field lines in a private flux zone, i.e. the field lines that do not enter the plasma and, therefore, have very short connection lengths.) Each helical stripe consists of layered basins of different poloidal turns $N_{\mathrm{p}}$. One should note that the distance between stripes of each pair depends on the plasma current: it increases with the plasma current [32]. It leads to the similar behaviour of the heat deposition patterns on the divertor target plates (see section 4.2).

\section{Transport of heat in the ergodic and laminar zones}

Several approaches to study the heat and the particle transport in the ergodic and the laminar zones have been developed. Below we describe ideas and obtained results from two methods applied to the TEXTOR-DED.

\subsection{The method of finite elements}

The first of these methods is a finite element code coupled with a finite difference method (FDM) for a three-dimensional transport simulation in the laminar zone. The idea of the modelling is based on the laminar zone.

The particles originating from the core region come to the edge almost following the field lines convectively, and they enter the finger. Because of its very thin structure, the particles are considered to diffuse easily to neighbouring regions with a short connection length of $1,2, \ldots$ turns, and then be guided to the wall along the field lines. It means that at the very edge close to the wall, the structure of relatively short connection length region is most likely responsible for the transport properties.

Considering such characteristics of laminar zones, one finds that a normal SOL model is still available in the analysis of transport there. That is, the short connection region is treated as a flux tube of the SOL, where the particles and heat flux from the core region are coming in through the long connection 

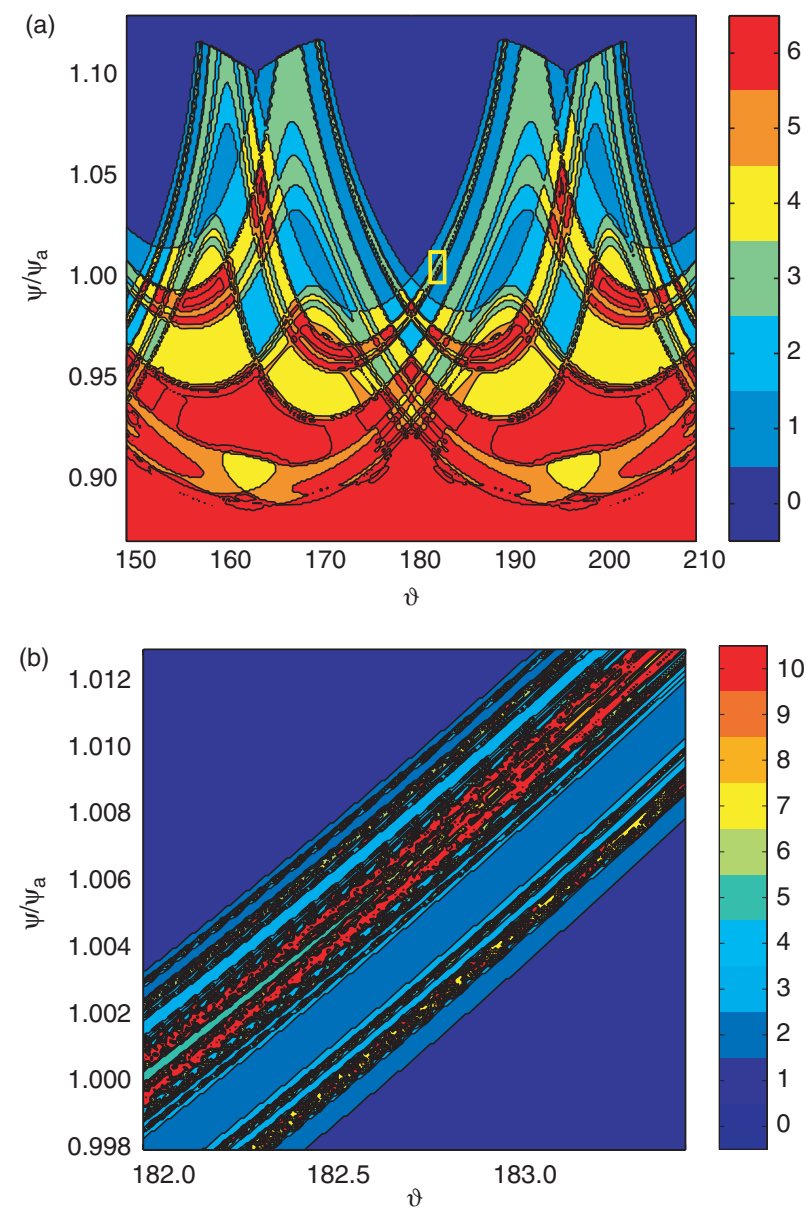

Figure 10. (a) Contour plot of $N_{\mathrm{p}}$ in the $\left(\vartheta, \psi / \psi_{\mathrm{a}}\right)$-plane corresponding to the Poincaré section in figure 8(c). (b) Expanded view of the rectangular area in $(a)$.

region or finger. In addition, because of the localization of the perturbation at the HFS, the majority of field lines hit the wall there. Therefore, in this representation, there are two locations (cuts) of the highest symmetry, a cut near the outer midplane and near the inner midplane as shown in figure 12(a). In these two cuts one expects stagnation points of the convective flow, namely, at the LFS for an odd number of poloidal turns and at the HFS for an even number of turns.

The first step with two-dimensional approximation has been done in [70-72], where the heat load patterns onto the plate were obtained. An attempt of the extension of the model to three-dimensional ones has been taken in [73, 74]. The numerical scheme is based on a splitting method, and consists of a FEM [79] for cross-field transport and a FDM for parallel transport [74]. At each time step, the FEM and the FDM are alternated. The cross-field transport is approximated on poloidal cross-sections at each $90^{\circ}$ toroidal angle, which is triangulated for the FEM. The triangles on each poloidal cut are connected in order to obtain volume cells for the FDM in the parallel direction.

One of the difficulties of the three-dimensional modelling is to construct a three-dimensional grid. Because of the perturbation field, the flux tube experiences a strong deformation while travelling around the torus. Figure 12(b) shows one of the examples for two poloidal turn region. One
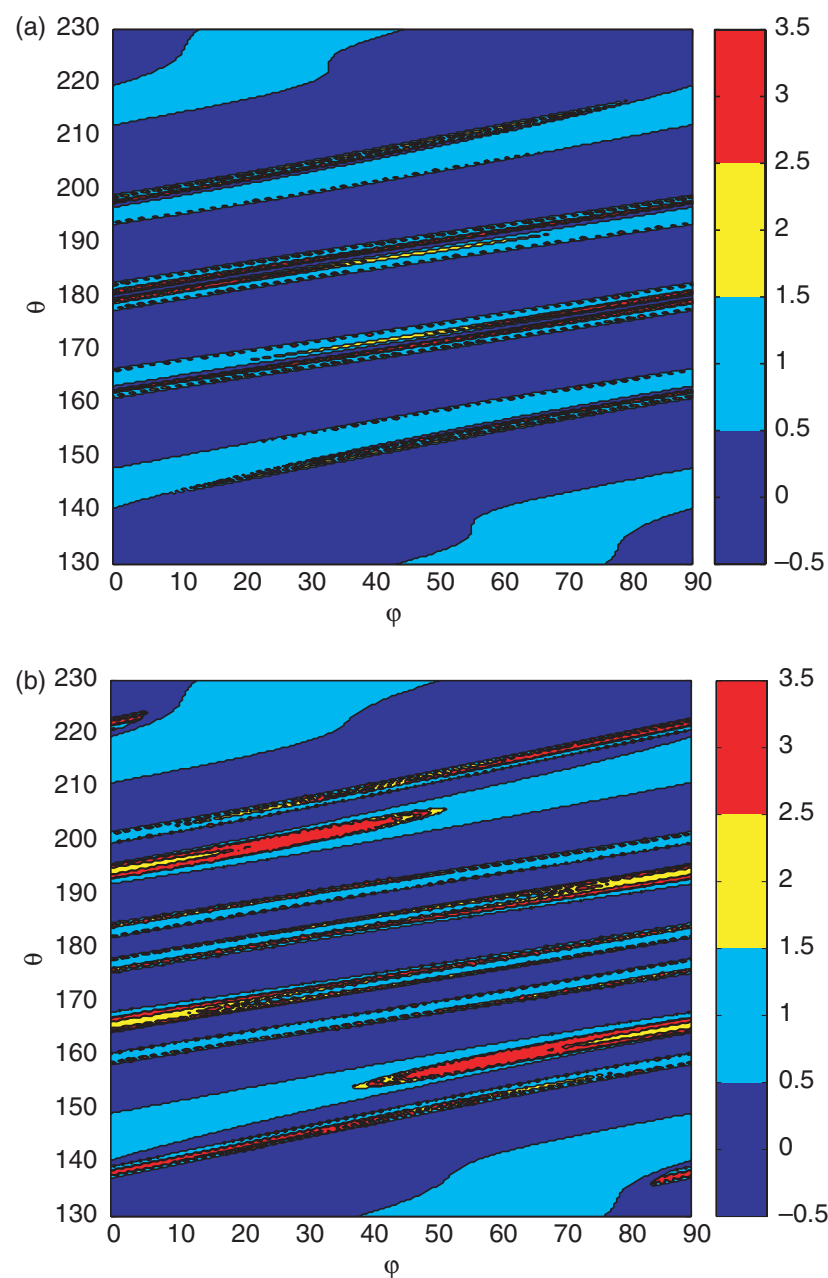

Figure 11. Basin boundary structure (magnetic footprints) on $\varphi, \theta$-plane on the divertor target plate. (a) For the plasma current $I_{\mathrm{p}}=460 \mathrm{kA} ;(b)$ for $I_{\mathrm{p}}=580 \mathrm{kA}$.

finds that especially in front of DED coils, i.e. HFS, the flux tubes experience the large deformation due to the localized perturbation field.

The flux tube is triangulated first at the stagnation point and those are mapped along the field lines to obtain the three-dimensional grid over the whole length of flux tube. The deformation, i.e. the direction of stretching, compression and bending, was carefully studied in order to obtain an optimized triangulation, in such a way that the area of each triangle is preserved as much as possible to be consistent with Hamiltonian description of the mapping.

The code was tested with a simple heat conduction problem, where the good agreement with analytical solution was obtained in [74]. Test results with a straight SOL configuration is described in [75].

\subsection{The Monte-Carlo method}

The most promising approach to the problem is the MonteCarlo method which has been used for the problem of heat balance in the DED in [76]. This method has been realized in a three-dimensional SOL plasma transport code E3D. This is a code under development to solve a system of plasma fluid 
(a)

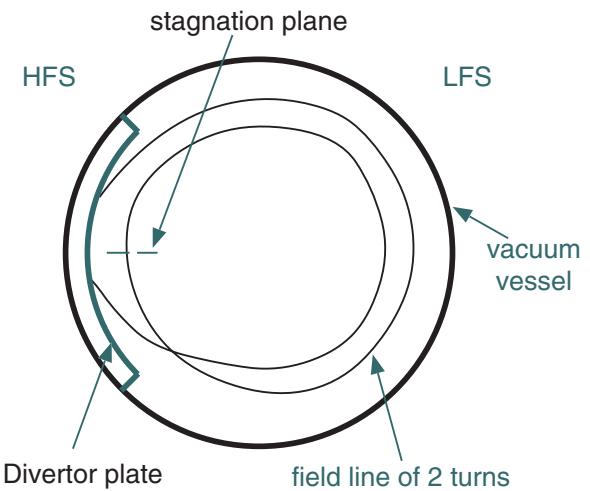

(b)

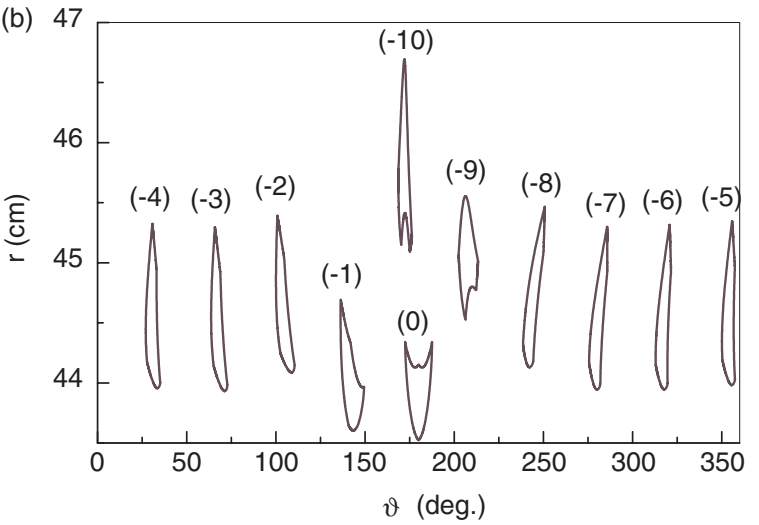

Figure 12. (a) An example of two poloidal turn field lines, where the trajectory is projected onto a certain poloidal cut. In this case the symmetric plane (stagnation plane) exits at HFS as shown with the broken line. For odd number of poloidal turns, the symmetric plane is located at LFS. (b) Flux tube of 2 poloidal turns. The 2 turn region at stagnation point $(0)$ was taken up from laminar plot, and mapped in counter clockwise in torus. Each flux tube in figure is apart by $90^{\circ}$ of toroidal angle from each other, those are projected onto the same poloidal plane.

equations in a general magnetic geometry. E3D was originally developed for tokamak ergodic divertors (TEXTOR-94). It was successfully benchmarked in a non-trivial case (single island geometry) against the three-dimensional finite-volume code BoRiS [77], and is currently being extended toward stellarator applications (W7-X).

The main idea of the Monte-Carlo method consists of the following: any fluid equation can be presented in the form of a conservation law of a certain quantity (internal energy in the case of heat balance equation). Therefore, the internal energy density can be interpreted as a density of 'energy parcels' which perform a random walk along and across the magnetic field. Within the Monte-Carlo procedure, the parameters of this random walk are chosen in such a way that the density of parcels satisfies on a long timescale the diffusion equation with the same parallel and perpendicular transport coefficients as those in the original heat balance equation. Such a method does not require a division of the edge region into flux tube areas with different poloidal turns, like the above method of finite elements. The method treats in the same way the partially chaotic ergodic zone with remnants of islands, the laminar zone and the regions with intact magnetic surfaces. Since parallel and perpendicular transport coefficients differ by a few orders of magnitude, special care must be taken to avoid the artificial numerical diffusion across the magnetic field in a general magnetic field topology. For this purpose, the multiple coordinate system approach (MCSA, see [76]) is used in E3D. This approach is based on the fact that magnetic field lines can exhibit stochastic behaviour only at distances which are large compared to the Kolmogorov length. This is the characteristic length of the exponential divergency of two initially neighbouring starting points. For smaller distances, the field lines remain regular. Thus, one can divide the computational domain into a finite set of sub-domains, introduce local magnetic coordinate systems in each and use an 'interpolated cell mapping' technique to change the coordinate systems.

Following [76], we restrict ourselves to the heat balance equation for electrons, neglecting convection for simplicity:

$$
\frac{\partial}{\partial t} \frac{3}{2} n T-\nabla \cdot\left[\kappa_{\perp} \nabla T+\left(\kappa_{\|}-\kappa_{\perp}\right) \boldsymbol{h h} \cdot \nabla T\right]=0 .
$$

Here $T$ stands for an electron temperature, $n$ is a plasma density (further assumed to be constant), $\boldsymbol{h}=\boldsymbol{B} / \boldsymbol{B}$ is a unit vector along the magnetic field. Here, we employ a phenomenological model of transport: the heat conduction parallel to a field line is classical (Braginskii), and the anomalous perpendicular transport is assumed to be a local heat conduction with a constant isotropic coefficient. Such a model assumes that parallel and perpendicular correlation lengths of the turbulence responsible for the cross-field transport are small compared to the corresponding scales of the magnetic field and plasma parameters. It is widely used in the divertor modelling (see, e.g. [80], chapter 4) allowing, in our case, the study of the 'pure' effect of the DED perturbation field on the plasma transport.

In general curvilinear coordinates $x^{i}$, equation (22) can be written in the following form,

$$
\frac{\partial T}{\partial t}=\frac{1}{\sqrt{g}} \frac{\partial}{\partial x^{i}} \sqrt{g}\left(D^{i j} \frac{\partial T}{\partial x^{j}}\right) .
$$

Here, $g$ is the metric determinant and $D^{i j}$ is the diffusion tensor appropriate for $T$,

$$
D^{i j}=\frac{2}{3}\left[\chi_{\perp} g^{i j}+\left(\chi_{\|}-\chi_{\perp}\right) h^{i} h^{j}\right],
$$

where $g^{i j}=\left(\nabla x^{i}\right) \cdot\left(\nabla x^{j}\right)$ and $h^{i}=\boldsymbol{h} \cdot \nabla x^{i}$ are contravariant components of the metric tensor and of the unit vector along the magnetic field, respectively, while $\chi_{\perp} \equiv \kappa_{\perp} / n$ and $\chi_{\|} \equiv \kappa_{\|} / n$. A strict separation of perpendicular and parallel heat fluxes (being different by several orders of magnitude for typical plasma parameters) is achieved in local magnetic coordinates where $h^{1}=h^{2}=0$. This is the type of coordinate system that is used in fusion plasma analysis to construct magnetic stream functions (Clebsch coordinates). To specify a particular local magnetic coordinate system, one should define some 'initial mesh' for the coordinates $x^{1}, x^{2}$. In general, these can be any two one-parametric families of curves on some surface which is never tangential to the magnetic field ('reference cut'). Extensions of these curves along the magnetic field lines form the coordinate surfaces $x^{1}=$ const. and $x^{2}=$ const. In [76] 
we used surfaces $\theta=$ const. as reference cuts with $r=$ const. and $\varphi=$ const. lines forming the 'initial mesh'. (Here $(r, \theta, \varphi)$ are quasitoroidal coordinates.) This choice has been optimized for stellarator applications.

With increasing distance from the reference cut the metric tensor of local magnetic coordinates becomes strongly anisotropic. If this distance becomes comparable with the Kolmogorov length, the coordinate surfaces $x^{1}=$ const. and $x^{2}=$ const. become almost parallel to each other. Therefore, we are forced to restrict the scope of a single coordinate system by some appropriate length which is smaller than the Kolmogorov length, hence the use of multiple coordinate systems. We couple the neighbouring coordinate systems with the help of an interpolated cell mapping technique. This is a precomputed transformation of the initial mesh $x^{1}=$ const., $x^{2}=$ const. projected along the magnetic field lines to a neighbouring reference cut, interpolated by means of bicubic splines. By increasing the mesh resolution within practically available computer memory limits, one can reduce the errors introduced by this kind of ICM to the level of the direct field line tracing error.

The Monte-Carlo method has been applied to both static and low-frequency dynamic problems (DC and $50 \mathrm{~Hz} \mathrm{AC}$ in the perturbation coils, respectively). Since plasma response has little effect on the perturbation magnetic field at low frequencies this field has been taken in vacuum approximation, as given by the GOURDON code. In addition, we can consider the low frequency dynamic regime as a sequence of quasistatic equilibria for different phasing of the perturbation coils. This is because the characteristic temperature relaxation time in SOL (of the order of $10 \mathrm{~ms}$ ) is much smaller than the oscillation period of the DED magnetic field.

The computational domain in the edge plasma of TEXTOR is presented in figure 13 in polar coordinates. We prescribe an input heat flux $250 \mathrm{~kW}$ in electron component from the core plasma and zero temperature on the wall, and use constant perpendicular heat conductivity $\chi_{\perp}=3 \mathrm{~m}^{2} \mathrm{~s}^{-1}$ and density $n=10^{13} \mathrm{~cm}^{-3}$.

The results presented in figures 13-15 demonstrate a strong effect of the perturbation magnetic field on the temperature distribution and, as a consequence, on the heat load pattern on the bumper limiter. A typical temperature profile at the plasma edge is shown in figure 13. It clearly shows that poloidal variation of the temperature field follows

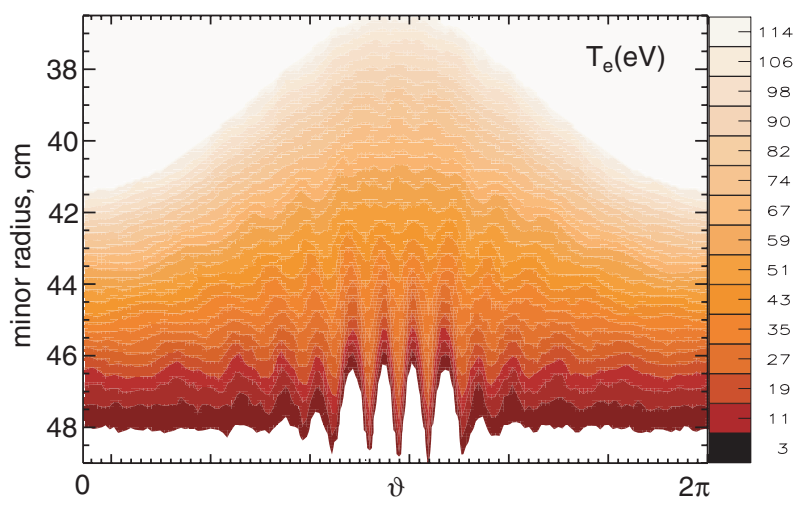

Figure 13. Temperature profile at the plasma edge. the structure of the magnetic field lines: hot 'fingers' here correspond to the regions with larger connection length indicated by more dense footprints in figure $8(a)$.

The heat load patterns on the bumper limiter in DC case are shown in figure 14 for three different plasma equilibrium currents: (a) $I_{\mathrm{p}}=460 \mathrm{kA}$, (b) $I_{\mathrm{p}}=520 \mathrm{kA}$, and (c) $I_{\mathrm{p}}=580 \mathrm{kA}$. Similarly to the magnetic footprints, the heat load is concentrated in four pairs of narrow stripes. The distance between the stripes in each pair increases with the plasma current (see section 3.4 and figure 11). The change in the phasing of the DED coils results predominantly in the toroidal shift of the stripes. This illustrates the idea of smearing the pattern away using the AC power supply in the perturbation coils (a dynamical operation mode). The variation
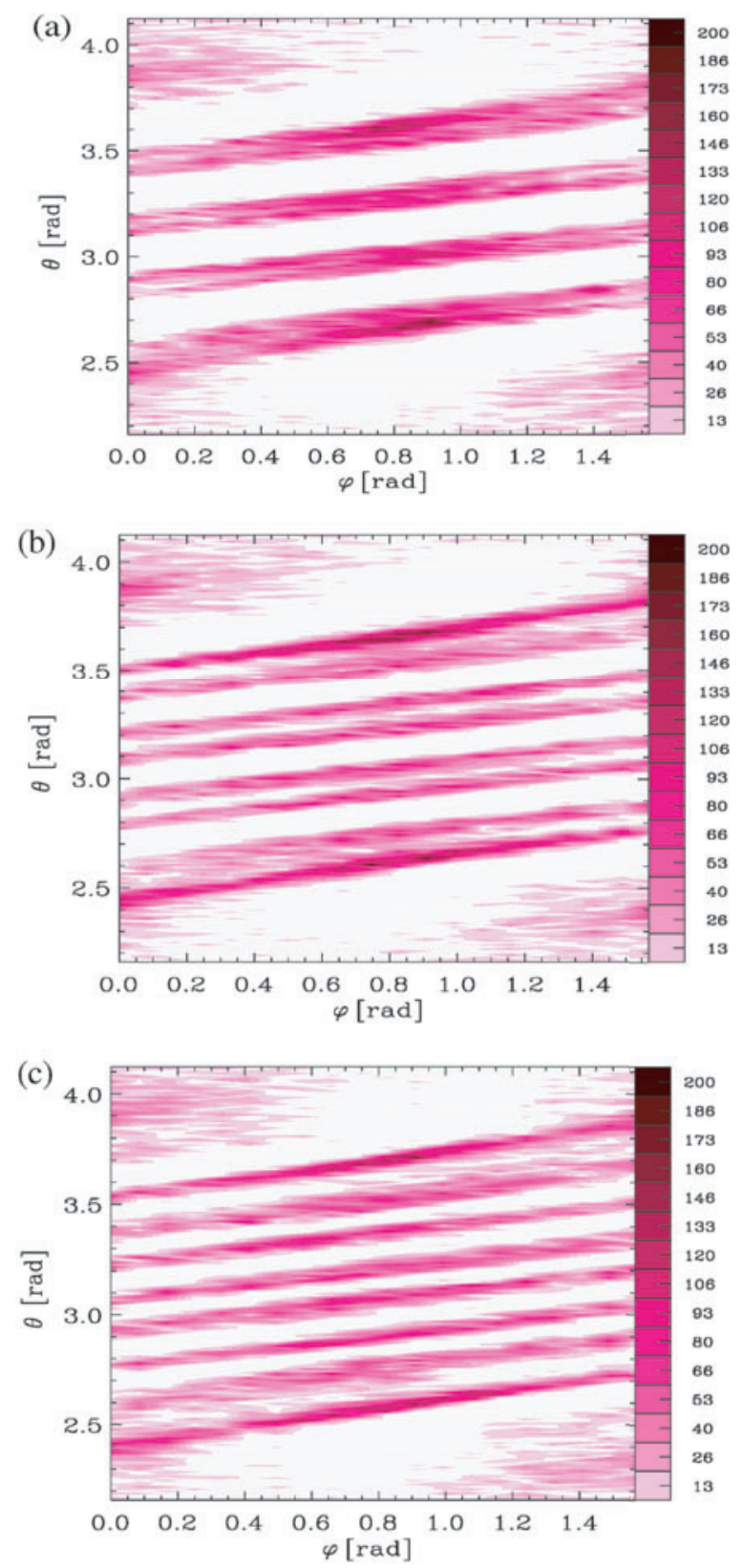

Figure 14. Power load onto the divertor target plates $\left(\mathrm{kW} \mathrm{m}^{-2}\right)$ depending on plasma current: $(a) I_{\mathrm{p}}=460 \mathrm{kA}$, (b) $I_{\mathrm{p}}=520 \mathrm{kA}$, (c) $I_{\mathrm{p}}=580 \mathrm{kA}$. 
(a)

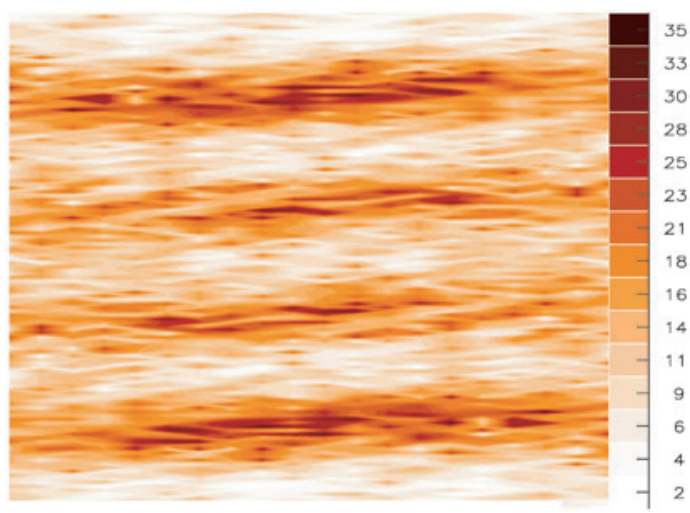

(b)

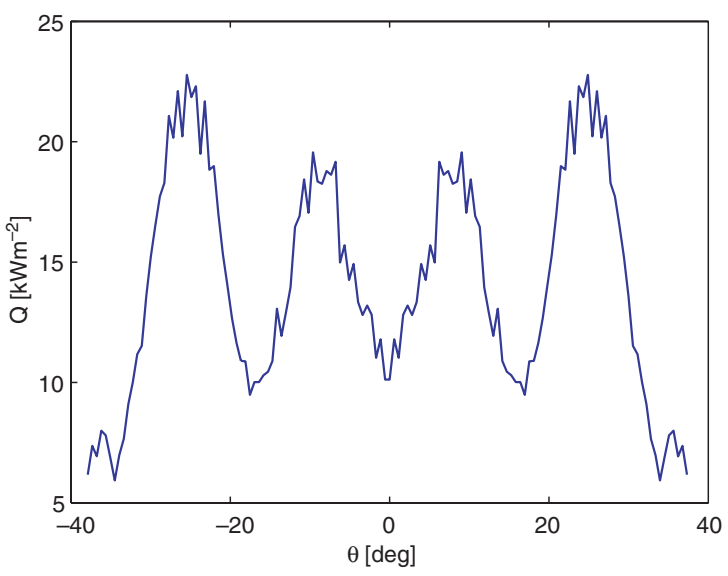

Figure 15. (a) Time averaged heat flux density on the divertor target plates. (b) The same, but averaged over toroidal angle.

of the magnetic field structure in time would result in a timevarying power load on the limiter such that the time-averaged heat load would have much smaller peak values than the heat load in the static operation mode. The effect of such time averaging is presented in figure 15. As one would expect, the average heat load pattern is almost toroidally symmetric, and the variation of the heat load with the poloidal angle is much smoother than in the static case. As a result, the peak power load appears to be reduced almost by one order of magnitude in this low-frequency dynamic regime.

\section{Conclusions}

In summary, we have reviewed the main results of the theoretical studies carried out during the last several years on the magnetic structure and heat transport at the stochastic plasma periphery induced by the DED which is now being installed for the TEXTOR. They are conditionally divided into three main topics: (i) methods of description of divertor coils, magnetic field and field line stochastization; (ii) a study of formation and control of the stochastic (ergodic) zone of field line, statistical properties of field lines; (iii) an investigation of heat and energy transport through the stochastic plasma periphery and the heat load patterns on the divertor target plates. The first topic included the description of the DED coils for the different operational regimes, features of the magnetic field created by these coils, the formulation of field line equations in Hamiltonian form using the magnetic (Boozer) coordinate system, and methods to study these equations. Particularly, a new method to integrate the Hamiltonian field line equations by symplectic mappings has been developed. The second topic is concerned with the problems of field line stochastization, formation and control of the ergodic and laminar zones by varying the plasma parameters, their statistical and fractal properties, magnetic footprints on the divertor target plates. And third, final topic discussed in the paper was related to transport studies during the DED operation. We have presented two main approaches to this problem: the FEM and the Monte-Carlo method to solve the heat balance equations at the plasma edge with stochastic field lines.

One should note that all three topics are related to the static $(\Omega=0)$ or low-frequency $(50 \mathrm{~Hz})$ operational regimes of the DED. Other important problems: the electric field generation at the plasma edge with chaotic field lines and the plasma behaviour in the high-frequency operational regimes $(1 \mathrm{kHz} \leqslant f \leqslant 10 \mathrm{kHz}$ ) of the DED were beyond the scope of this review. The variation of the electric field has both global and fine scale character, and it has been discussed on TEXT and Tore Supra [11].

The high-frequency operation of the DED introduces a new element into the ergodic divertor concept. There are two important issues that arise in this regime of the DED: the penetration of the rotating external magnetic field into the plasma and the possible plasma rotation induced by this DED field. The first issue has been recently experimentally studied in the small research tokamak CSTN at Nagoya University with a rotating magnetic field $[81,82]$. The first theoretical studies of the penetration of the high-frequency external magnetic field into the plasma in the DED has been carried out in [83-85]. Particularly, in [85] the possibility of the induced plasma rotation has been discussed. First results from nonlinear modelling by a reduced MHD code of a cylindrical plasma model indicates that the external DED field couples at the outermost plasma layer to a skin current, even though the electrical conductivity of the edge plasma is very low because of very low electron temperature. Without friction, the edge plasma is quickly accelerated to the wave velocity and the DED field penetrates quickly into the plasma. With the assumption of a friction, a slippage between wave field and plasma persists and the skin current remains at a moderate level. Between the skin layer and the resonance layer, the current density is low; at the resonant layer the main dissipation of energy from the DED field and consequently the main part of the force transfer from the external currents to the plasma takes place. The excited shielding current depends again critically on the assumptions of plasma acceleration and friction. However, it is expected that in toroidal direction a force will be created with about the same value as given by the tangential NBI ( $\sim 2 \mathrm{MW}$ unidirectional) which results in a substantial plasma rotation; in poloidal direction, the force is even an order of magnitude larger but also the neoclassical frictional force. Poloidal rotations are therefore only expected in the layer around the resonant zones. The differential rotation (e.g. due to the torque transfer from NBI and the DED) in the plasma edge may provide a new knob to influence the turbulence and the plasma transport.

Beginning of operation of the TEXTOR-DED will allow us to study experimentally not only the classical problem of 
ergodic divertor, but also its new features related with a rotating magnetic field.

\section{References}

[1] Engelhardt W. and Feneberg W. 1978 J. Nucl. Mater. 76 \& 77518

[2] Feneberg W. and Wolf G.H. 1981 Nucl. Fusion 21669

[3] Samain A., Grosman A. and Feneberg W. 1982 J. Nucl. Mater 111 \& 112408

[4] Finken K.H. (ed) 1997 Dynamic Ergodic Divertor (special issue) Fusion Eng. Design 37 335-448

[5] Gentle K.W. 1981 Nucl. Technol. Fusion 1479

[6] McCool S.C. et al 1989 Nucl. Fusion 29547

[7] Deschamps P., Grosman A., Lipa M. and Samain A. 1984 J. Nucl. Mater. 128 \& 12938

[8] Samain A., Grosman A., Blenski T., Fuchs G. and Steffen B. 1984 J. Nucl. Mater. 128 \& 129395

[9] Lipa M., Aymar R., Deschamps P., Hertout P., Portafaix C. and Samain A. 1989 Fusion Technology: Proc. 15th Symp. (Utrecht, 1988) (Amsterdam: Elsevier) p 874

[10] Nguyen F., Ghendrih Ph. and Samain A. 1995 Calculation of magnetic field line topology of ergodized zone in real tokamak geometry. Application to the tokamak TORE SUPRA through the MASTOC code Rep. DRFC/CAD EUR-CEA-FC-1539 CEA Cadarache, St Paul-lez-Durance

[11] Ghendrih Ph., Grossman A. and Capes H. 1996 Plasma Phys. Control. Fusion 381653

[12] Nguyen F., Ghendrih P. and Grossman A. 1997 Nucl. Fusion 37743

[13] Grossman A. 1999 Plasma Phys. Control. Fusion 41 A185

[14] Kawamura T., Abe Y. and Tazima T. 1982 J. Nucl. Mater. $111 \& 112268$

[15] Finken K.H. et al 1995 J. Nucl. Mater. 220-222 448

[16] Nicolai A. 1997 Fusion Eng. Design 37347

[17] Kaleck A., Hassler M. and Evans T. 1997 Fusion Eng. Design 37353

[18] Ghendrih Ph., Grosman A. and Kaleck A. 1997 Proc. 24th European Physical Society Conf. on Control. Fusion and Plasma Physics (Berchtesgaden, 1997) vol 21A (Europhysics Conference Abstracts) ed M. Schittenhelm et al (Petit-Lancy: The European Physical Society) p 1685

[19] Kaleck A., Finken K.H. and Ha $\beta$ ler M. 1997 Proc. 24th European Physical Society Conf. on Control. Fusion and Plasma Physics (Berchtesgaden, 1997) vol 21A (Europhysics Conference Abstracts) ed M. Schittenhelm et al (Petit-Lancy: The European Physical Society) p 1689

[20] Finken K.H. 1997 Fusion Eng. Design 37379

[21] Finken K.H. 1997 Nucl. Fusion 37583

[22] Finken K.H. 1998 Trans. Fusion Technol. 33291

[23] Abdullaev S.S., Finken K.H., Kaleck A. and Spatschek K.H. 1998 Phys. Plasmas 5196

[24] Finken K.H., Eich Th. and Kaleck A. 1998 Nucl. Fusion 38515

[25] Fischer O. and Cooper W.A. 1998 Plasma Phys. Rep. 24727

[26] Eich T., Finken K.H. and Kaleck A. 1998 Contrib. Plasma Phys. 38112

[27] Abdullaev S.S., Finken K.H., Kaleck A., Spatschek K.H. and Wolf G. 1998 Czech. J. Phys. 48 (suppl S2) 319

[28] Abdullaev S.S., Finken K.H. and Spatschek K.H. 1999 Phys. Plasmas 6153

[29] Kaleck A. 1999 Contrib. Plasma Phys. 39367

[30] Finken K.H., Abdullaev S.S., Kaleck A. and Wolf G. 1999 Nucl. Fusion 39637

[31] Finken K.H. et al $1999 \mathrm{~J}$. Nucl. Mater. 266-269 495

[32] Abdullaev S.S., Eich Th. and Finken K.H. 2001 Phys. Plasmas 82739

[33] Kobayashi M., Eich Th., Abdullaev S.S. and Finken K.H. 2002 IEEE Trans. Plasma Phys. 3066

[34] Abdullaev S.S. 1999 J. Phys. A 322745

[35] Abdullaev S.S. 2002 J. Phys. A 352811
[36] Kerst D.W. 1962 J. Nucl. Energy 4C 253

[37] Rosenbluth M.N., Sagdeev R.Z., Taylor J.B. and Zaslavsky G.M. 1966 Nucl. Fusion 6297

[38] Filonenko N.N., Sagdeev R.Z. and Zaslavsky G.M. 1967 Nucl. Fusion 7253

[39] Boozer A.H. 1981 Phys. Fluids 291999

[40] Cary J.R. and Littlejohn R.G. 1983 Ann. Phys. (NY) 1511

[41] Chirikov B.V. 1979 Phys. Rep. 52265

[42] Lichtenberg A.J. and Lieberman M.A. 1992 Regular and Stochastic Motion 2nd edn (New York: Springer)

[43] Martin T.J. and Taylor J.B. 1984 Plasma Phys. Control. Fusion 26321

[44] Regianni N. and Sakanaka P.H. 1994 Plasma Phys. Control. Fusion 36513

[45] Viana R.L. and Caldas I.L. 1992 Z. Naturforsch. A 47941

[46] Caldas I.L., Pereira J.M., Ullmann K. and Viana R.L. 1996 Chaos, Solitons and Fractals 7991

[47] Ullmann K. and Caldas I.L. 2000 Chaos, Solitons and Fractals 112129

[48] Feron S. and Ghendrih Ph. 1997 Proc. 24th European Physical Society Conf. on Control. Fusion and Plasma Physics (Berchtesgaden, 1997) vol 21A (Europhysics Conference Abstracts) ed M. Schittenhelm et al (Petit-Lancy: The European Physical Society) (part 1) p 185

[49] Da Silva E.C., Caldas I.L. and Viana R.L. 2001 Phys. Plasmas 82855

[50] Greene J.M. 1984 Statistical Physics and Chaos in Fusion Plasmas ed C.W. Horton Jr and L.E. Reichl (New York: Wiley) pp 3-20

[51] Mendonca J.T. 1991 Phys. Fluids B 387

[52] Wobig H. 1987 Z. Naturforsch. A 421054

[53] Wobig H. and Fowler R.H. 1988 Plasma Phys. Control. Fusion 30721

[54] Bazzani A., Malavasi M. and Siboni S. 1989 Il Nuovo Cimento 103659

[55] Bazzani A., Di Sebastiano A. and Turchetti G. 1998 Il Nuovo Cimento D 201795

[56] Punjabi A., Verma A. and Boozer A. 1992 Phys. Rev. Lett. 69 3322

[57] Punjabi A., Verma A. and Boozer A. 1994 J. Plasma Phys. 5291

[58] Punjabi A., Verma A. and Boozer A. 1996 J. Plasma Phys. 56569

[59] Punjabi A., Ali H. and Boozer A. 1997 Phys. Plasmas 4337

[60] Abdullaev S.S. and Zaslavsky G.M. 1995 Phys. Plasmas 2 4533

[61] Abdullaev S.S. and Zaslavsky G.M. 1996 Phys. Plasmas 3516

[62] Abdullaev S.S. and Finken K.H. 1998 Nucl. Fusion 38531

[63] Rochester A.B. and Rosenbluth M.N. 1978 Phys. Rev. Lett. 4038

[64] Yamagishi T., Hinton F.L., Bhadra D.K. and Miller R.L. 1983 Nucl. Fusion 23189

[65] Samain A., Capes H., Ghendrih Ph. and Nguyen F. 1993 Phys. Fluids B 5471

[66] Ghendrih Ph., Capes H., Nguyen F. and Samain A. 1992 Contrib. Plasma Phys. 32179

[67] Becoulet M., Ghendrih Ph., Capes H. and Grosman A. 1999 Europhysics Conf. Abstracts 23J 989

[68] Becoulet M., Ghendrih Ph., Capes H. and Grosman A. 1999 Electron heat transport in stochastic magnetic layer Rep. EUR-CEA-FC-1667 CEA Cadarache, St Paul-lez-Durance, p 28

[69] Becoulet M., Capes H., Ghendrih Ph., Grosman A., Gunn J., Hoang G.T., Segul J.L. and Zabiego M. 2000 Contrib. Plasma Phys. 40251

[70] Finken K.H., Eich Th. and Kaleck A. 1998 Nucl. Fusion 38515

[71] Eich T., Reiser D. and Finken K.H. 2000 Nucl. Fusion 401757

[72] Eich T. and Finken K.H. 2000 Contrib. Plasma Phys. 4057

[73] Eich T., Reiser D. and Finken K.H. 2001 J. Nucl. Mater. 290-293 849 
[74] Kobayashi M., Sewell G., Finken K.H., Eich Th., Reiser D. and Abdullaev S.S. 2002 Contrib. Plasma Phys. 42163

[75] Kobayashi M., Reiser D., Sewell G., Finken K.H. and Abdullaev S.S. 2003 J. Nucl. Mater. 313-316 1061

[76] Runov A.M., Reiter D., Kasilov S.V., Heyn M.F. and Kernbicher W. 2001 Phys. Plasmas 8916

[77] Runov A., Kasilov S., Riemann J., Borchardt M., Reiter D. and Schneider R. 2002 Contrib. Plasma Phys. 42169

[78] Ott E. and Tel T. 1993 Chaos 3417

[79] Sewell G. 1988 The Numerical Solutions of Ordinary and Partial Differential Equations (New York: Academic)
[80] Stambaugh R. et al 1999 ITER Physics Basis (special issue) Nucl. Fusion 39 2391-469

[81] Kobayashi M., Tuda T., Tashiro K., Kojima H., Zhai K. and Takamura S. 2000 Nucl. Fusion 40181

[82] Kobayashi M., Kojima H., Zhai K. and Takamura S. 2000 Phys. Plasmas $\mathbf{7} 3288$

[83] Faulconer D.W. and Koch R. 1977 Fusion Eng. Des. 37399

[84] Finken K.H. 1999 Nucl. Fusion 39707

[85] Finken K.H., Abdullaev S.S., Eich T., Faulconer D.W., Kobayashi M., Koch R., Mank G. and Rogister A. 2001 Nucl. Fusion 41503 